\title{
Excesso de Incentivos à Inovação na Presença de Consumidores Sofisticados. Um Modelo de Progresso Tecnológico Endógeno com Capital Humano
}

\author{
- aurora A. C. TeiXeira*
}

\section{RESUMO}

Neste artigo é desenvolvido um modelo de crescimento baseado no progresso tecnológico endógeno, em que o crescimento econômico é induzido por melhorias contínuas na qualidade de cada produto diferenciado. A inovação, "motor" do crescimento, tem como "combustível" essencial o capital humano. Partindo dos modelos de base de Grossman e Helpman (I99/a, I99Ib), mas considerando, diferentemente, um índice de consumo constituído por bens diferenciados e um bem homogêneo, o modelo proposto destaca a influência determinante da procura no crescimento econômico, aspecto negligenciado pela literatura do crescimento endógeno. Do esforço de modelização decorre que consumidores pouco sofisticados, com reduzida preferência por produtos diferenciados em termos de qualidade, geram, seguramente, incentivos insuficientes para a investigação, enquanto que consumidores mais sofisticados podem gerar excesso de incentivos. Assim, se a quota dos produtos diferenciados na procura estiver positivamente correlacionada com o nível de desenvolvimento do país, então países menos desenvolvidos tendem a apresentar taxas de inovação relativamente reduzidas, ao contrário de países mais desenvolvidos, em que essas taxas podem até ser (socialmente) demasiado elevadas.

\section{PALAVRAS-CHAVE}

modelos de crescimento, Pesquisa \& Desenvolvimento, capital humano

\section{ABSTRACT}

In this article it is developed a growth model based on endogenous technological progress, where growth is induced by continuous improvements in the quality of each differentiated product. The innovation, the "engine" of growth, has human capital as its critical fuel. Based on Grossman \& Helpman's (I99 Ia, 199 Ib) models but differently considering an index of consumption constituted by both differentiated goods and a homogenous good, the model proposed highlights the crucial influence of demand on economic growth, an issue neglected by endogenous growth literature. From the modeling effort it results that less sophisticated consumers, with reduced preferences by quality differentiated products generate, for sure, insufficient incentives to research, whereas highly sophisticated consumers might generate excessive incentives. Thus, in the case the share of differentiated products is correlated with the country's development level, less developed countries tend to present relatively low innovation rates, contrasting with more developed countries, where these rates might be (socially) excessive.

\section{KEY WORDS}

growth models, Research \& Development, human capital

\section{JEL CLASSIFICATION}

O4I, 032, J24

\footnotetext{
- A autora agradece os valiosos e incisivos comentários de dois pareceristas anônimos. Quaisquer erros e omissões remanescentes são de exclusiva responsabilidade da autora. Agradece, igualmente, o suporte de pesquisa do CEMPRE - Centro de Estudos Macroeconómicos e Previsão - apoiado pela Fundação para a Ciência e a Tecnologia, Portugal, por meio do Programa Operacional Ciência, Tecnologia e Inovação (POCTI) do Quadro Comunitário de Apoio III, que é financiado pelo FEDER e fundos portugueses.

* Professora da Faculdade de Economia, Universidade do Porto e investigadora do CEMPRE e INESC Porto. Endereço para contato: Faculdade de Economia do Porto, Rua Dr. Roberto Frias, 4200-464, Porto, Portugal. E-mail: ateixeira@fep.up.pt.

(Recebido em julho de 2005. Aceito para publicação em agosto de 2006).
} 


\section{INTRODUÇÃO}

Um dos fatos mais extraordinários da dinâmica das economias capitalistas ao longo dos últimos séculos é a emergência contínua de novas alternativas de consumo que acompanham o crescimento da renda e da produtividade em economias em expansão. (Fatas-Villafranca e Saura-Bacaicoa, 2004). Longe de satisfazer os consumidores, o crescimento econômico exponencial parece ter estimulado os desejos humanos oferecendo novidade e variedade incorporadas num incessante fluxo de atrativos bens. (Grossman e Helpman, 1991b; Bianchi, 2002). Embora isto seja um fato bem conhecido que caracteriza a evolução capitalista, pouca atenção tem sido atribuída pela teoria moderna do crescimento à compreensão do fenômeno do lado da procura, associado à relevância crescente das atividades de consumo na sociedade. ${ }^{1}$

$\mathrm{Na}$ verdade, a teoria ortodoxa do crescimento econômico, quer na sua forma neoclássica inicial (Solow, 1956), quer na nova ou endógena teoria do crescimento (ver Barro e Sala-i-Martin, 1995, para uma survey), negligencia a procura agregada. (Dutt, 2006). Na teoria ortodoxa do crescimento econômico o foco reside tradicionalmente na lógica da acumulação do capital e na influência das diferentes formas de alterações técnicas. No entanto, embora a tecnologia, a produção e a oferta sejam elementos críticos para a capacidade de uma economia crescer, isto não constitui toda a história. Historicamente, tem existido e continuam a existir alterações maciças nos produtos e serviços oferecidos à procura final, assim como alteraçôes no comportamento dos consumidores e nos padrões de consumo ao longo do processo de crescimento econômico. (Witt, 2001a).

Alguns autores de correntes mais heterodoxas, nomeadamente evolucionistas (e.g., Metcalfe, 2001; Saviotti, 2001; Witt, 2001a,b), argumentam que o que acontece precisamente do lado da procura constitui uma parte essencial da teoria do crescimento econômico. De acordo com esta perspectiva, o papel do consumidor como "inovador" tem sido profundamente subestimado nas abordagens schumpeterianas da inovação e crescimento, principalmente do lado da oferta. (Metcalfe, 2001).

Tentando requalificar o papel-chave da procura no processo de crescimento econômico, e assim reconciliar, em certa medida, as duas abordagens teóricas do crescimento (ortodoxa e evolucionista) acima referidas, o presente artigo tem como objetivo principal apresentar um modelo de crescimento endógeno, em que o crescimento é induzido por melhorias na qualidade dos produtos. Partindo dos modelos-base de Grossman e Helpman (1991a, 1991b), mas de forma distinta destes, e considerando um índice de

1 Uma exceção importante a esta falta de interesse no papel do consumo no crescimento econômico é a contribuição de Pasinetti $(1981,1993)$. Este autor defende que qualquer investigação sobre o progresso tecnológico deveria incluir hipóteses sobre a evolução da procura (e, especificamente, a procura por bens de consumo) relativamente ao crescimento da renda. 
consumo constituído por bens diferenciados e um bem homogêneo, o modelo proposto destaca a influência determinante da procura, atribuindo aos consumidores a importância que estes detêm no mundo real.

Um resultado inovador do modelo desenvovido no presente artigo aponta para o fato de, na eventualidade de o peso dos bens diferenciados no índice do consumo ser relativamente baixo, a taxa de equilíbrio de mercado se situar sempre aquém da que seria socialmente desejável (ótima), independentemente da dimensão dos avanços tecnológicos. Adicionalmente, quando os consumidores apresentam um consumo relativamente enviesado em direção aos bens diferenciados em face do mais elevado montante de lucros proporcionado por uma maior procura de bens diferenciados, existe a possibilidade de ocorrerem situações em que a taxa de equilíbrio de mercado gera excesso de incentivos em termos de bem-estar, ou seja, é superior à que seria socialmente adequada.

O presente artigo está estruturado da seguinte forma. Na seção a seguir, o modelo é descrito de forma sumária. Na seção 2, desenvolve-se a estrutura analítica do mesmo, procurando determinar a taxa de inovação de equilíbrio de mercado e a taxa de crescimento do índice de consumo. Pelo fato de se estar na presença de um modelo que envolve estruturas de mercado de concorrência imperfeita (para os bens qualitativamente diferenciados, o preço de mercado é superior ao custo unitário de produção) e spillovers de conhecimento que implicam rendimentos crescentes de escala, analisamse as questões de bem-estar (seção 3). Concretamente, a taxa de crescimento ótima é determinada, e a partir da comparação desta última com a taxa de crescimento de mercado, são tecidas considerações quanto à insuficiência ou excesso de incentivos à $P \& D$. Finalmente, o artigo é concluído com uma síntese dos principais resultados do modelo. Para facilitar a fluência da leitura, remete-se ao Apêndice diversos passos técnicos da derivação dos resultados do modelo.

\section{BREVE DESCRIÇÃO DO MODELO}

A composição do sistema econômico mudou profundamente ao longo do tempo. (Saviotti, 2001). A constatação de que tem havido inúmeras alterações qualitativas ao longo do tempo é um fato inegável para qualquer economista. (Saviotti, 1988, 1991, 1994, 1996). As economias modernas contêm um grande número de entidades (produtos, serviços, metódos de produção, competências, atores individuais e organizacionais, instituições), que são qualitativamente novas e diferentes comparativamente às que existiam em sistemas econômicos passados. (Saviotti e Mani, 1995). De fato, o desenvolvimento e o crescimento econômico dependem da capacidade de o sistema econômico criar novos (ou novas versões de) bens e serviços. No entanto, 
para que contribuam para o crescimento econômico, tais bens e serviços terão que ser adquiridos pelos consumidores. A dinâmica do desenvolvimento da procura constitui, assim, um determinante fundamental do desenvolvimento econômico. (Saviotti, 2001; Sonobe, Hu e Otsuka, 2004).

Tendo por base esta intuição empírica, no modelo teórico descrito nesta seção a economia cresce devido à contínua melhoria de um conjunto de produtos diferenciados, que dá origem a um acréscimo no bem-estar dos consumidores. A utilidade/nível de satisfação dos consumidores depende de um índice de consumo agregado constituído por um número fixo de produtos diferenciados, daí o aumento do nível de satisfação ser determinado exclusivamente pelas melhorias na qualidade de cada um desses produtos.

A opção por esta abordagem do crescimento econômico, via inovação dos produtos, baseada em esforços das empresas especificamente orientados para tentar a melhoria da qualidade dos mesmos, prende-se não apenas ao fato deste enquadramento ser atualmente aquele que, em termos teóricos, constitui uma formulação mais completa dos fundamentos microeconômicos do fenômeno agregado, que é o crescimento econômico (e por isso mais aderente à complexidade dos comportamentos dos agentes econômicos que estão subjacentes às relações econômicas), mas resulta também (e, talvez, sobretudo) da constatação empírica de que grande parte dos esforços de pesquisa por parte das empresas são, hoje em dia, destinados a melhorias dos produtos já existentes no mercado, constituindo as inovações radicais (novos produtos ou novos processos) um fenômeno menos freqüente. ${ }^{2}$ Nesta mesma linha de opção, introduz-se o fator capital humano, procurando tornar evidente a sua importância no processo de inovação de um país e, como tal, no respetivo crescimento, bem patente nas extraordinárias performances de economias como a Coréia do Sul e Taiwan, caracterizadas por grandes investimentos em capital humano. (Collins, 1990).

A estrutura e pressupostos utilizados baseiam-se essencialmente nos modelos de Aghion e Howitt (1992), Grossman e Helpman (1991a, 1991b) e Segerstrom (1991). Em particular, segue-se de perto o tipo de exposição feita por Grossman e Helpman (1991b). Trata-se aqui de uma dupla diferenciação em nível dos produtos. Existe um conjunto fixo de produtos diferenciados que entram na cesta de consumo de cada indivíduo (diferenciação horizontal), estando cada um deles disponível num número ilimi-

2 Segundo um estudo do Gabinete de Estudos e Planejamento do Ministério da Indústria e Energia (GEPIE, 1992) de Abril de 1992, baseado numa pesquisa com 3.276 estabelecimentos industriais ( $25 \%$ das empresas das indústrias extrativa e de transformação com mais de 10 pessoas empregadas) para o período 1987-1989, a melhoria dos produtos existentes constituía a inovação de produto mais frequiente $(69,1 \%)$, seguida da introdução de vários novos produtos e de um novo produto, com $26,8 \%$ e $15,2 \%$, respectivamente. Vale, no entanto, esclarecer que tais porcentagens tendem a subestimar a importância da diferenciação vertical, já que muitos dos novos produtos vêm substituir produtos que desempenhavam funções semelhantes. 
tado de qualidades distintas (diferenciação vertical). É a dinâmica inerente à evolução da qualidade de cada um dos produtos que potencializa o crescimento econômico. ${ }^{3}$ A taxa de crescimento econômico depende da composição, dimensão e alocação dos recursos disponíveis em cada momento do tempo e, em particular, do capital humano afeto à pesquisa que produz as novas qualidades de cada produto diferenciado.

Tal como em Grossman e Helpman (1991a, 1991b) e em Aghion e Howitt (1992), a Pesquisa e Desenvolvimento (P\&D) é considerada uma atividade essencial, cujo sucesso potencializa as melhorias na qualidade dos produtos existentes (logo, do crescimento econômico). Sendo o capital humano o input fundamental da atividade de $\mathrm{P} \& \mathrm{D}$, ele constitui o recurso acumulável crítico ao processo de crescimento. Na linha de Romer (1990), o capital humano é a "variável-escala" do crescimento econômico. Uma economia com capital humano escasso tem um setor de pesquisa inexpressivo e, portanto, pouco apto a produzir melhorias na qualidade dos produtos e, conseqüentemente, incapaz de gerar crescimento econômico.

Seguindo Schultz (1961), Becker, Murphy e Tamura (1990), Romer (1990), Grossman e Helpman (1991b) e Barro (1991), o capital humano é a acumulação de esforço destinado à escolaridade e à aprendizagem. Considera-se que este capital é, em cada momento do tempo, constante, negligenciando os efeitos cumulativos inerentes a este fator. ${ }^{4}$ Este pressuposto, aparentemente extremo, se justifica pelo fato de o modelo ser de progresso tecnológico. Aqui, o interesse centra-se na relação capital humano $\Rightarrow$ progresso tecnológico, e não o contrário.

Ao contrário de Grossman e Helpman (1991b) e Romer (1987), não se considera a força de trabalho como o input base da atividade de pesquisa. Embora seja plausível que uma economia com maior quantidade de trabalho realize mais $\mathrm{P} \& \mathrm{D}$, gerando, por isso, uma maior taxa de inovação de produto, a sua consideração implicaria que as economias de maior dimensão (com uma maior quantidade de mão-de-obra/população) tenderiam, ceteris paribus, a crescer mais rapidamente, resultado este que se pretende evitar. Em geral, o crescimento mais rápido apenas se verifica se se aumentar a quantidade dos fatores que as atividades potenciadoras do crescimento econômico (no presente modelo, a P\&D) utilizam mais intensivamente. Assim, o capital humano constitui, neste modelo, a medida correta da escala de uma economia e não a população. (Romer, 1990).

3 Grossman e Helpman (1992, cap.3) e Romer (1990) apresentam modelos semelhantes ao aqui desenvolvido, mas em que o crescimento econômico está baseado no incremento do número de produtos diferenciados.

4 Embora, num horizonte de vida finita, o capital humano de um indivíduo não possa crescer ilimitadamente, as qualificações que o indivíduo adquire podem ser aplicadas a um conjunto cada vez mais apurado de tecnologias de produção, daí o fato de o valor desse capital continuar a crescer ao longo do tempo e com ele o ritmo de crescimento. É este efeito cumulativo que, por assunção, é negligenciado. 
A literatura teórica recente sobre a pesquisa industrial como motor endógeno do crescimento centra-se em dois conceitos fundamentais. Um primeiro conceito traduz o fato de as empresas, maximizadoras do lucro, procurarem alcançar poder de mercado por meio da produção de um bem (de qualidade) superior ao dos seus mais diretos rivais. Neste contexto, ao longo do tempo, bens e serviços mais novos substituem os mais antigos, usufruindo de lucros temporários, e sendo posteriormente também substituídos. Há aqui implícita a idéia daquilo que Aghion e Howitt (1992) designam, seguindo Schumpeter (1934), de "efeito de destruição criativa", que, simultaneamente, incentiva e limita o valor privado da inovação industrial. O segundo conceito aponta para a natureza de "bem público" do conhecimento que potencializa rendimentos crescentes de escala para a economia como um todo. Os spillovers tecnológicos desempenham aqui um papel fundamental, quando uma empresa (inovadora) coloca no mercado um novo produto, possibilitando, desta forma, aos investigadores (potenciais inovadores) a obtenção de informações sobre a tecnologia de produção e atributos do novo produto. Assim, os rivais poderão iniciar esforços de pesquisa com o intuito de melhorar o produto "estado-de-arte", mesmo que não tenham sido bem-sucedidos anteriormente na produção do mesmo. Nas palavras de Caballero e Jaffe (1993, p.16), "[i]n the process of creating new goods, inventors rely and build on the insights embodied in previous ideas; they achieve their success partly by 'standing upon the shoulders of giants." Desta forma, as invenções contribuem para o conhecimento público, e este mesmo conhecimento facilita a inovação subseqüente.

Neste contexto, as empresas (inovadoras) investem recursos com a esperança de descobrir algo com valor comercial, isto é, esperam ser capazes de retirar um lucro positivo dos seus esforços de pesquisa. Assim, tais empresas têm que ser capazes de vender os seus produtos a preços que excedam os respectivos custos unitários, de modo a recuperar os seus investimentos iniciais na pesquisa. Ou seja, "... some imperfect competition in product markets is necessary to support private investments in new technologies." (Grossman e Helpman, 1994, p. 32).

O processo de inovação "à Schumpeter" traduz o fato do inovador bem-sucedido (o mais recente) destronar o líder anterior, apropriando-se da parte dos lucros deste na indústria do produto em questão.

A modelização do processo de crescimento em nível microeconêmico tem subjacente que aquele processo é desigual e estocástico. As empresas concorrem entre si para lançar a próxima geração do produto, podendo existir, numa determinada indústria, longos períodos em que não ocorre qualquer sucesso, ao passo que outras indústrias podem experimentar sucessivas e rápidas investigações bem-sucedidas. Não obstante, em nível macroeconômico, a agregação dilui, em parte, esta turbulência microeconômica - a economia como um todo, considerando a existência de um elevado número 
de melhorias na qualidade do conjunto (fixo) de produtos, cresce a um ritmo estacionário. Assim, são os custos e benefícios da pesquisa que determinam o ritmo de crescimento de longo prazo.

\section{ESTRUTURA DO MODELO-BASE}

\subsection{Consideraçôes Iniciais}

Considera-se aqui um contínuo de produtos diferenciados, indexados por $k$, em que $k$ $\in[0, N]$, sendo $N$ constante. Por simplificação, normalizamos $N$ a 1 .

Cada produto diferenciado $k$ tem os seus degraus de qualidade ("quality ladder"), podendo ser oferecido em um número ilimitado de qualidades.

Cada inovação é construída a partir da anterior, ou seja, quando uma empresa de pesquisa alcança um avanço tecnológico (melhoria do produto) na linha do produto $k$, o produto estado-de-arte naquela indústria progride uma geração. ${ }^{5}$

Seja $q_{j}(k) \equiv$ qualidade da geração $j$ do produto $k(j=0,1,2, \ldots)$.

Cada nova geração do produto fornece $\lambda$ vezes mais serviços que o produto da geração anterior $q_{j}(k)=\lambda q_{j-1}(k) \forall j, k$, em que $\lambda>1$ é exógeno, constante e comum a todos os produtos ( $\lambda \equiv$ dimensão da inovação).

As unidades são escolhidas de modo a que a qualidade mais baixa de cada produto (aquela que estava disponível na geração 0 ) ofereça uma unidade de serviço, $q_{0}(k)=1$. Donde se retira, $q_{j}(k)=\lambda^{j} \forall j, k, \lambda>1$.

Definimos o produto estado-de-arte como o produto disponível mais recentemente inventado (ou seja, o de qualidade mais elevada), em cada momento do tempo.

Os empresários, em cada momento do tempo, colocam os seus esforços sobre um único produto e concorrem entre si para produzir a próxima geração desse produto. Assim, os empresários que, para cada produto, têm capacidade de produzir o produto estado-de-arte ou alguma geração anterior daquele, concorrem entre si como oligopolistas. Desta concorrência surge um fluxo de lucro, que representa a recompensa para as empresas que foram bem-sucedidas na atividade de pesquisa. Neste contexto, os esforços de pesquisa bem-sucedidos incentivam novas melhorias, levando os inves-

5 Daí a expressão “... 'standing upon the shoulders of giants" de Newton, citada por Caballero e Jaffe (1993, p. 16). 
tidores a concorrerem incessantemente para colocar no mercado a próxima geração do produto em questão.

Decorre daí que a distribuição da qualidade dos produtos evolui ascendentemente ao longo do tempo (e com esta o bem-estar dos agentes econômicos), seguindo cada produto uma progressão estocástica de sequiências de qualidade. Apesar desta aleatoriedade em nível da indústria, em termos agregados o processo é uniforme. No steady-state, como se verificará, o índice de consumo cresce a uma taxa constante e determinada.

Os consumidores, por sua vez, têm à sua disposição um conjunto de bens diferenciados (representados pelo vector $\mathbf{X}=\left[\begin{array}{llll}\mathbf{x}_{1} & \mathbf{x}_{2} & \ldots & \mathbf{x}_{\mathrm{k}}\end{array}\right]$ ) e um bem homogêneo, representado pelo escalar $Y$ - numerário ${ }^{6}$-, cujo preço é, por definição, unitário.

O mercado do bem homogêneo, $Y$, é de concorrência perfeita, ao passo que o dos bens diferenciados, $\mathbf{X}$, se caracteriza por uma concorrência de Bertrand.

Existem dois fatores de produção, trabalho não-qualificado $(L)$ e estoque de capital humano $(H)$. Relativamente à intensidade de utilização dos fatores, assume-se que a atividade de $\mathrm{P} \& \mathrm{D}$ e a produção dos bens diferenciados, $\mathbf{X}$, empregam unicamente capital humano, enquanto que a produção do bem homogêneo exige capital humano e trabalho não-qualificado (fator específico de $Y$ ).

Dado que se pretende enfatizar aqui a importância do capital humano no crescimento econômico, enquadrado num modelo de progresso tecnológico endógeno, negligenciam-se aspectos importantes como o crescimento da população e a da oferta de trabalho, evitando, desta forma, as questões sobre a fertilidade, participação na força de trabalho e variação das horas de trabalho. Considera-se também que o estoque de capital humano na população é exógeno (isto é, dado em cada momento do tempo), assim como a fração deste fornecida ao mercado. Assim, as ofertas agregadas de estoque de capital humano, $H$, e de trabalho, $L$, são fixas. Este modelo é, pois, de crescimento endógeno em relação ao progresso tecnológico, e não ao capital humano.

\subsection{Preferências dos Consumidores}

As preferências dos consumidores são análogas às propostas em Grossman e Helpman (1991b), com a consideração adicional do bem homogêneo. Os consumidores da economia oferecem uma quantidade fixa de trabalho não-qualificado e capital humano em troca de um salário, recebem juros sobre ativos, compram bens para consumir

6 Os preços de $\mathbf{X}$ são relativos ao produto homogêneo $Y$. 
e poupam por meio da acumulação de ativos adicionais. Ao efetuar os seus planos, os consumidores levam em conta o bem-estar dos seus descendentes (Barro, 1974), razão pela qual a presente geração maximiza a utilidade intertemporal num horizonte temporal infinito.

Assim, o consumidor representativo desta economia maximiza a utilidade total, $U$, dada pela seguinte função utilidade intertemporal: ${ }^{7}$

$$
\begin{aligned}
U_{t} & =\int_{0}^{\infty} e^{-\rho t} C(t) d t \\
U_{t} & =\int_{0}^{\infty} e^{-\rho t}[\alpha \log u(t)+(1-\alpha) \log Y(t)] d t
\end{aligned}
$$

em que

$\rho$ : taxa de desconto intertemporal, $\rho>0 ;{ }^{8}$

$\alpha$ : fração da utilidade dos bens diferenciados na utilidade total do indivíduo;

$u(t)$ : consumo agregado dos produtos diferenciados, no momento $t$;

$y(t)$ : consumo do bem homogêneo, no momento $t$;

$u(t)^{\alpha} y(t)^{1-\alpha}$ : índice de consumo, no momento $t$.

A utilidade instantânea relativa ao consumo agregado de $X$, para cada indivíduo, é dada por:

$$
\alpha \log u(t)=\alpha \int_{0}^{1} \log \left(\sum_{j} q_{j}(k) d_{j t}(k)\right) d k
$$

em que

$q_{j}(k)$ : qualidade da geração $j$ do produto $k$;

$d_{j t}(k)$ : consumo da geração $j$ do produto $k$, no momento $t$.

7 Especifica-se uma função utilidade logarítmica, que constitui um caso especial da função utilidade mais geral, $U_{t}=\int_{0}^{\infty} e^{-\alpha} \frac{C(t)^{1-\sigma}-1}{1-\sigma} d t, \sigma>0$, em que a elasticidade de substituição intertemporal é unitária $(\sigma=1)$. Apesar desta última especificação permitir um intervalo mais amplo de elasticidades de substituição, a maior generalidade que daqui se retira contribui muito pouco para a análise, razão pela qual optou-se por sacrificar a generalidade em favor da simplicidade.

8 Um valor positivo para $\rho$ significa que a utilidade é tanto menos valorizada quanto mais tarde for recebida. Ramsey (1928) assume $\rho=0$, considerando o agente maximizador mais um planejador social do que um consumidor que escolhe o consumo e a poupança, quer da geração atual, quer das geraçốes futuras. 
O somatório em (2) engloba o conjunto das geraçóes do produto $k$ que estão disponíveis no momento $t$. É de se notar que a qualidade, $q_{j}$, contribui positivamente para a utilidade.

Os consumidores estão sujeitos a uma restrição orçamentária intertemporal dada por:

$$
\int_{0}^{\infty} e^{-R(t)}[E(t)+Y(t)] d t \leq A(0)
$$

em que

$E(t)+Y(t)$ : despesa total, no momento $t$;

$R(t)$ : fator do juro acumulado até ao momento $t, R(t)=\int_{0}^{t} r(\tau) d \tau$;

$A(0)$ : valor atual do fluxo dos rendimentos dos fatores, mais os ativos detidos inicialmente pelo indivíduo.

Por sua vez, $E(t)$ é a despesa total em produtos diferenciados e $Y(t)$ é a despesa no bem homogêneo, dadas, respectivamente, por

$$
\begin{aligned}
& E(t)=\int_{0}^{1}\left(\sum_{j} p_{j t}(k) d_{j t}(k)\right) d k \\
& Y(t)=y(t) p_{Y}(t)=y(t) \text { dado que } p_{Y}(t)=1
\end{aligned}
$$

em que

$p_{j t}(k)$ : preço de uma unidade da geração $j$, do produto $k$, no momento $t$;

$d_{j t}(k)$ : consumo da geração $j$ do produto $k$, no momento $t$;

$p_{Y}(t)$ : preço do bem homogêneo, $Y$ (numerário), no momento $t$.

O objetivo do consumidor representativo é maximizar (1), respeitando a restrição orçamentária (3). Admitindo que se trata de bens normais, com elasticidade-renda unitária, este processo de otimização do consumidor pode ser (por conveniência analítica e facilidade de exposição) separado em três fases:

1) dada a despesa agregada em bens diferenciados num dado momento do tempo, $E(t)$, determinar a alocação ótima desta entre esses mesmos bens;

2) dada a despesa total num dado momento do tempo, $E(t)+Y(t)$, determinar a distribuição ótima entre $E(t)$ e $Y(t)$; 
3) considerando a alocação da despesa que maximiza a utilidade do consumidor em cada momento do tempo (quer relativa às quotas dos produtos diferenciados em $E(t)$, quer às quotas de $E(t)$ e $Y(t)$ na despesa total), determinar a distribuição de $E(t)$ ao longo do tempo de forma a maximizar as preferências intertemporais.

No que respeita à alocação estática da despesa agregada dos bens diferenciados, o consumidor maximiza a utilidade instantânea, selecionando para cada um desses bens uma única geração $j$, cujo preço, ajustado pela qualidade, é o mais baixo. Ou seja, seleciona $J_{t}(k)$, tal que $p_{J t} / q_{J_{t}}=\min \left\{p_{j t}(k) / q_{j}(k) ; j=0,1, \ldots \infty\right\}$.

Tal procedimento dá origem às funções procura estática para os produtos diferenciados,

$$
d_{j t}(k)=\left\{\begin{array}{ccc}
\frac{E(t)}{p_{j t}(k)}, & \text { para } & j=J_{t}(k) \\
0, & \text { para } & j \neq J_{t}(k)
\end{array}\right.
$$

Em seqüência, a utilidade instantânea relativa aos produtos diferenciados é dada por:

$$
\begin{aligned}
& \alpha \log u(t)=\alpha \int_{0}^{1}\left(\log \sum_{j} q_{j}(k) \frac{E(t)}{p_{j t}(k)}\right) d k, \text { ou, de outra forma } \\
& \alpha \log u(t)=\alpha \int_{0}^{1}\left(\log \sum_{j} \frac{q_{j}(k)}{p_{j t}(k)}+\log E(t)\right) d k
\end{aligned}
$$

Como, para cada produto (e supondo o preço uniforme), o consumidor seleciona apenas a qualidade líder (ou seja, o produto estado-de-arte), então:

$$
\begin{aligned}
& \alpha \log u(t)=\alpha \log E(t)+\alpha \int_{0}^{1}\left(\log \frac{q_{t}(k)}{p_{t}(k)}\right) d k \\
& \alpha \log u(t)=\alpha \log E(t)+\alpha \int_{0}^{1}\left(\log q_{t}(k)-\log p_{t}(k)\right) d k .
\end{aligned}
$$

Para maximizar a sua utilidade instantânea global (englobando, para além dos produtos diferenciados, o consumo do bem homogêneo) o consumidor distribui a despesa 
total $(E+Y)$ proporcionalmente entre bens diferenciados e o bem homogêneo (ver Apêndice):

$$
\frac{\alpha}{1-\alpha}=\frac{E(t)}{Y(t)}
$$

Desta forma, as expressões da utilidade e da restrição orçamentária do consumidor representativo são as seguintes:

$$
\begin{aligned}
U_{t}= & \int_{0}^{\infty} e^{-\rho t}\left[\log E(t)+\alpha \int_{0}^{1}\left(\log q_{t}(k)-\log p_{t}(k)\right) d k+(1-\alpha) \log \left(\frac{1-\alpha}{\alpha}\right)\right] d t \\
& \frac{1}{\alpha} \int_{0}^{\infty} e^{-R(t)} E(t) d t \leq A(0)
\end{aligned}
$$

A solução para o problema dinâmico do consumidor consiste na determinação do máximo da função utilidade intertemporal (l') sujeito à restrição orçamentária (3'), $\underset{\{E(t)\}}{\operatorname{Max}} U_{t} \quad$ s.a. $\frac{1}{\alpha} \int_{0}^{\infty} e^{-R(t)} E(t) d t \leq A(0)$. A solução é a seguinte trajetória temporal ótima dos gastos (ver Apêndice):

$$
\frac{\dot{E}}{E}=r-\rho
$$

em que $r$ é a taxa de juro, em cada momento do tempo, e $\dot{E}=\frac{d E}{d t}$.

\subsection{Lado da Oferta-Produtores}

Como referido no início da presente seção, nesta economia existem dois fatores de produção: trabalho não-qualificado, $L$, e capital humano, $H$. Admite-se também, dada a ênfase no capital humano, um caso particular relativo à intensidade de utilização dos fatores em cada um dos setores considerados: os setores de P\&D e dos produtos diferenciados utilizam exclusivamente capital humano, enquanto que o setor do bem homogêneo utiliza capital humano e trabalho não-qualificado (input específico a este setor).

9 Idêntica solução se usará, doravante, para fazer referência à derivada de uma variável em relação ao tempo $(t)$. 
Para as atividades de pesquisa e produção de bens diferenciados admite-se tecnologias de coeficientes fixos. Assim, na atividade de pesquisa, uma unidade de P\&D exige $a_{H I}$ unidades de capital humano, enquanto que na produção dos bens diferenciados uma unidade deste tipo de bem exige $a_{H X}$ unidades de capital humano.

No que tange à produção do bem homogêneo, $Y$, considera-se uma tecnologia neoclássica exibindo rendimentos constantes de escala e substituibilidade entre os inputs $(L, H)$ necessários à produção.

\subsubsection{Induistria do Bem Homogêneo}

Como a estrutura de mercado para o bem homogêneo, $Y$, é de concorrência perfeita, e todo o trabalho não-qualificado é empregado na sua produção, os produtores deste bem, maximizadores de lucros, produzem uma quantidade de $Y$ tal que $p_{r}=c_{Y}\left(\mathfrak{w}_{H}, L\right)$, em que $c_{Y}($.$) : custo marginal de produção do bem Y$; $p_{Y}$ : preço do bem $Y$ (dado); $\mathfrak{w}_{H}$ : preço de uma unidade de capital humano. Como o bem $Y$ é o numerário, $I=c_{Y}\left(w_{H}, L\right)$.

\subsubsection{Indústria dos Produtos Diferenciados}

Seguiu-se nesta subseção a derivação do equilíbrio feita por Grossman e Helpman (1991a, 1991b), assumindo que todas as empresas concorrem nos preços. Existem leis sobre as patentes que protegem indefinidamente os direitos de propriedade intelectual dos inovadores (proporcionando à empresa inovadora o direito exclusivo de vender os bens que inventa) e não é possível o licenciamento de patentes. Desta forma, garante-se que toda a produção de bens diferenciados é levada a cabo por empresas que desenvolveram, com sucesso, os novos produtos estado-de-arte.

No sentido de descrever o processo de fixação de preços nesta indústria, imaginemos duas empresas distintas: uma (líder) tem acesso à tecnologia do produto estado-dearte, enquanto a outra (seguidora) é capaz de produzir o bem que se posiciona, em termos qualitativos, um degrau abaixo do produto líder.

\section{$1^{a}$ Situação}

Num determinado momento, a empresa seguidora fixa o preço, $\mathbf{c}_{\mathrm{X}}$, que é o preço mais baixo consistente com lucros não negativos.

Os consumidores estão dispostos a pagar um prêmio por um produto estado-de-arte, no entanto, optarão pelo produto da geração imediatamente anterior se o líder fixar um preço que exceda $\lambda c_{X}$ ( $\lambda$ : acréscimo de serviços/qualidade proporcionado pelo bem líder relativamente ao produto do seu rival mais próximo). 


\section{GRÁFICO 1 - CURVA DA PROCURA PERCEBIDA PELO LÍDER DA INDÚSTRIA}

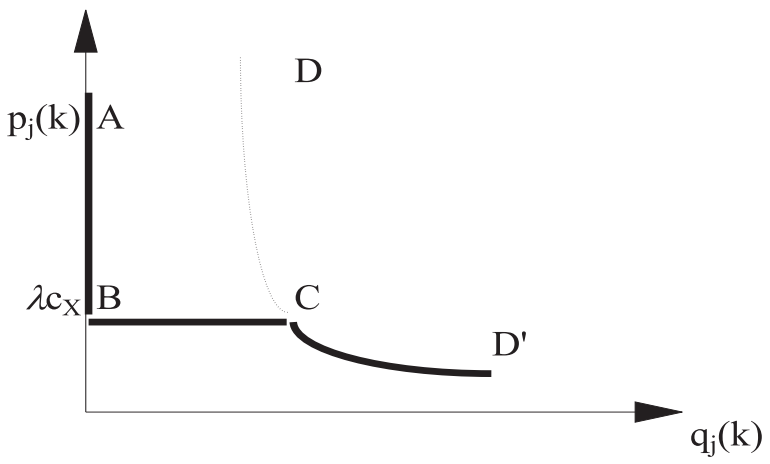

Fonte: Grossman e Helpman (1993, p. 90).

Quando o líder fixa um preço inferior a $\lambda c_{X}$, toda a procura da indústria passa a ser satisfeita por si, pois o seu produto oferece um preço (por unidade de qualidade) inferior à do seguidor. Se os preços fixados pelo líder e pelo seguidor forem, respectivamente, $\lambda c_{X} \mathrm{e} c_{X}$, então o líder pode vender qualquer quantidade ao longo do segmento BC. Desta forma, a resposta ótima do líder, diante do preço $c_{X}$ fixado pelo seu rival mais próximo, é estabelecer um preço infinitesimalmente abaixo de $\lambda c_{X}$. Isto se justifica por três razões: 1) com esse "preço limite" afasta a outra empresa do mercado; 2) se fixar um preço superior a $\lambda c_{X}$, perde os clientes em favor da empresa seguidora; 3) se fixar um preço discretamente inferior a $\lambda c_{x}$, não está sendo racional, porque as funções-procura da indústria têm elasticidade unitária, não permitindo ganhos na receita marginal. Adotando tal comportamento, o líder absorve toda a procura da indústria ficando o mais próximo do preço de monopólio que a indústria concorrente permite. ${ }^{10}$

\section{$2^{a}$ Situação}

Quando o líder fixa o preço $\lambda c_{X}$ a resposta ótima por parte da empresa seguidora consistirá em fixar o preço $c_{X}$, por duas ordens de razão: 1) se diminuir o preço (fixando-o abaixo do custo marginal), incorrerá em perdas; 2) se o aumentar, colocar-se-á numa posição de indiferença, pois nesta situação as suas vendas serão nulas.

O equilíbrio de Bertrand-Nash é atingido se o líder praticar o preço "preço-limite", expulsando o seu mais direto rival (seguidor) do mercado, como explicam Grossman e Helpman (1993).

10 Barro e Sala-i-Martin (1995, cap. 7) apresentam uma análise detalhada das diferenças entre preço-limite e preço de monopólio, no contexto deste tipo de modelo. 
Como este equilíbrio pode ser estendido ao caso mais geral em que a liderança tecnológica pode ultrapassar uma geração $\left(\lambda^{j}\right)$, conclui-se que o produto estado-de-arte tem sempre o preço (ajustado da qualidade) mais reduzido.

Com as hipóteses já adiantadas sobre a tecnologia do desenvolvimento do produto e sobre a natureza dos direitos de propriedade garante-se que em cada indústria e em cada momento existe apenas um líder, estando este sempre um degrau à frente do seu rival mais próximo (qualquer que seja a indústria em questão).

Do exposto, resulta que todos os produtos estado-de-arte (líderes de qualidade) de cada indústria têm o mesmo preço-limite $[\mathbf{p}(\mathrm{k})=\mathbf{p}]:^{11}$

$$
\mathbf{p}=\lambda \mathbf{c}_{\mathbf{x}}
$$

em que

p: vector linha $(1 \times k)$ de preços idênticos para os $k$ produtos diferenciados;

$\mathbf{c}_{X}$ : vector linha $(1 \times k)$ de custos marginais idênticos para os $k$ produtos diferenciados.

Substituindo (10) em (6), obtemos as seguintes funções-procura:

$$
d_{j t}=\left\{\begin{array}{lll}
\frac{E(t)}{p}=\frac{E(t)}{\lambda c_{x}}, & \text { se } & j=J_{t}(k) \\
0 & , \text { se } & j \neq J_{t}(k)
\end{array}\right.
$$

O fluxo de lucro do monopolista consiste em:

$$
\pi=\mathbf{p} X-\mathbf{c}_{X} X=\mathbf{p} X-\frac{\mathbf{p}}{\lambda} X=\left(1-\frac{1}{\lambda}\right) \mathbf{p} X=\left(1-\frac{1}{\lambda}\right) E
$$

Em equilíbrio, os líderes não investem quaisquer recursos para melhorar os seus próprios produtos estado-de-arte, donde todas as inovações são realizadas pelos seguidores que, quando bem-sucedidos, ficam um degrau à frente do líder anterior. ${ }^{12}$

11 Trata-se de produtos diferenciados, com idêntico custo marginal.

12 Um líder prefere ter os seus recursos afetados no desenvolvimento de uma posição de liderança num outro mercado do que ampliar o seu avanço (em termos de qualidade) no mercado onde atualmente se encontra. Isto porque o acréscimo de lucro que daí retiraria $-\Delta \pi=\left(1-\frac{1}{\lambda^{2}}\right) E-\left(1-\frac{1}{\lambda}\right) E=\left(1-\frac{1}{\lambda}\right) \frac{E}{\lambda}$ 
Vale salientar, no entanto, que a P\&D é uma atividade com risco. Uma empresa pode dirigir todos os seus esforços de pesquisa para um qualquer produto estado-de-arte. Se esta empresa investir recursos em P\&D a uma intensidade $i$ durante um intervalo de tempo de duração $d t$, tem uma probabilidade $i d t$ de sucesso no desenvolvimento da próxima geração do produto. Assim, o sucesso na atividade de P\&D envolve uma distribuição de probabilidade de Poisson, com uma taxa de ocorrência que depende do nível atual da atividade de P\&D. Para alcançar uma intensidade $i$ de P\&D a empresa tem que investir $a_{H I} i$ unidades de capital humano, por unidade de tempo. Desta forma, a probabilidade de sucesso da empresa na pesquisa é estritamente proporcional aos recursos investidos (capital humano).

Os produtores potenciais podem, analisando os produtos estado-de-arte disponíveis no mercado, obter preciosas informações técnicas que lhes permitem realizar os seus próprios esforços de pesquisa. ${ }^{13}$ Quando o produtor potencial (empresa seguidora) é bem-sucedido(a) no seu esforço de pesquisa, ele(a) toma a liderança da indústria para o produto selecionado e usufrui de um fluxo de lucro, dado por (12). Estes lucros cessam assim que o próximo sucesso de pesquisa seja alcançado (por outra empresa) na mesma linha de produto.

O fluxo de lucros na equação (12) é idêntico para todas as indústrias $k$. Assim, desde que a duração esperada da liderança seja também idêntica, as empresas são indiferentes quanto à indústria para a qual destinará os seus esforços de pesquisa. Tal como Grossman e Helpman (1991b, 1993), considera-se um equilíbrio simétrico aquele em que todos os produtos estão sujeitos à mesma intensidade agregada de P\&D (i), razão pela qual essa indiferença se verifica. ${ }^{14}$

\section{Condição de Livre-entrada}

Consideremos $v$ o valor atualizado do lucro (incerto) que fluirá para o líder da indústria, i.e, o valor de mercado da empresa líder ou, de forma equivalente, o montante do prêmio proporcionado pelo sucesso em P\&D.

Uma empresa poderá obter $v$ com uma probabilidade $i d t$, investindo um montante de recursos $a_{H i} i$ durante um intervalo de tempo $d t$ incorrendo num custo $w_{H} a_{H I} i d t$.

- é estritamente menor que o acréscimo de lucro que um não líder obteria se fosse bem-sucedido no seu esforço de investigação, isto é, $\left(1-\frac{1}{\lambda}\right) E$.

13 Isto traduz os já mencionados benefícios de spillovers da inovação, que estão ligados à natureza de bem público da tecnologia.

14 Como os investigadores esperam que as inovações futuras tenham igual probabilidade nas diferentes indústrias (isto é, que o fluxo de lucros tenha igual duração em cada indústria), eles ficam indiferentes quanto à indústria a selecionar. 
O financiamento da empresa é realizado via emissão de títulos, os quais, no caso do esforço de pesquisa ser bem-sucedido, atribuem aos seus titulares o fluxo de rendimento associado ao líder da indústria. Se, pelo contrário, o esforço de pesquisa falhar, os detentores dos títulos incorrem numa perda equivalente ao total do capital investido, v. Os detentores de títulos podem, no entanto, neutralizar o risco, detendo carteiras bem diversificadas. Para tal, é exigido apenas que cada uma das empresas maximize o ganho líquido esperado dos respectivos esforços de pesquisa, vidt $-w_{H} a_{H l} i d t$.

A nova empresa deve, pois, escolher a sua intensidade de pesquisa de modo a maximizar o benefício líquido esperado dessa mesma pesquisa. Deste modo, sempre que o valor presente do lucro, $v$, for inferior ao custo do investimento na pesquisa, $a_{H I} w_{H}$, não ocorre qualquer investimento $(i=0)$. Em contrapartida, este investimento tende para o infinito quando $v>a_{H I} w_{H}$.

Num equilíbrio com um investimento em pesquisa positivo, mas finito, verifica-se $v=a_{H I} w_{H}$, caso em que as empresas de pesquisa ficam indiferentes quanto à escala dos seus esforços de pesquisa. Desta forma, a condição de livre-entrada no setor da P\&D é:

$$
a_{H I} w_{H} \geq v \quad \text { com igualdade sempre que } i>0
$$

Os líderes da indústria geram um fluxo de dividendos de $\pi d t$ durante o intervalo de duração $d t$. Se nenhum dos esforços de pesquisa rivais for bem-sucedido durante aquele período de tempo, os acionistas das empresas líderes usufruem de ganhos de capital no montante de $\dot{v} d t$. Ou seja, os acionistas obtêm um ganho de capital de $\dot{v} d t$ com probabilidade (1-idt), admitindo que os esforços de pesquisa das empresas em questão são estatisticamente independentes. Mas o produto líder pode ser melhorado durante o intervalo $d t$, com uma probabilidade $i d t$. Neste último caso, os acionistas sofrem perdas no montante do capital total investido, $v$. Desta forma, a taxa de retorno esperada das ações da empresa, por unidade de tempo, é $\frac{\pi+\dot{v}}{v}-i$.

Como os resultados de pesquisa nas diferentes indústrias não estão contemporaneamente correlacionados, os riscos que o líder enfrenta são idiossincrásicos, razão pela qual os acionistas podem obter um rendimento certo se detiverem uma carteira bem diversificada de ações de cada empresa nas diferentes indústrias. ${ }^{15}$

O mercado tende então a valorizar a empresa para que a taxa de retorno das suas ações seja exatamente igual à taxa de juro sobre as obrigaçóes sem risco, $r$.

15 Trata-se aqui de ausência de correlação estatística entre a possibilidade de sucesso dos vários esforços de investigação contemporâneos, o que não põe em questão a existência de spillovers. 


$$
\frac{\pi+\dot{v}}{v}-i=r
$$

A equação anterior representa a condição de não-arbitragem.

\subsection{Equilibrio dos Mercados de Trabalbo e de Bens}

Conforme referido anteriormente, uma unidade de pesquisa exige $a_{H I}$ unidades de capital humano. Assim, o emprego total deste fator na atividade de pesquisa é igual a $a_{H I} i$.

Na produção dos bens diferenciados, cada unidade de output requer $a_{H X}$ unidades de capital humano. Por simplificação, mas sem perda de generalidade, assumimos $a_{H X}=1$.

A procura agregada de capital humano efetuada pelos produtores destes bens é representada por $\frac{E(t)}{\lambda c_{X}}$, pois cada indústria do produto diferenciado produz $\frac{E(t)}{p}=\frac{E(t)}{\lambda c_{X}}$ [veja-se (11)], e a dimensão do setor está normalizada por construção $(k \in[0,1])$, dado que a produção destes bens utiliza exclusivamente, como imput, capital humano, $c_{X}=w_{H}$.

A indústria do bem homogêneo, $Y$, para produzir uma unidade de produto, necessita de capital humano, $H$, e trabalho não-qualificado, $L$. O Lema de Shephard implica que a procura de capital humano e trabalho não-qualificado por parte desta indústria seja, respectivamente, $a_{H Y}\left(w_{H}, w_{L}\right)$ e $a_{L Y}\left(w_{H}, w_{L}\right)$ por unidade de produto, em que $a_{j Y}($.$) é o$ requisito de fator $j(j=H, L)$ na produção de $Y$, que iguala a derivada parcial de $c_{Y}($. em relação a $w_{j}$.

Em termos genéricos, as condições de equilíbrio no mercado dos fatores são $a_{H I} i+a_{H X} X+a_{H Y}\left(w_{H}, w_{L}\right) Y=H \quad$ e $\quad a_{L Y}\left(w_{H}, w_{L}\right) Y=L$. No caso concreto:

$$
\begin{aligned}
& a_{H I} i+\frac{E(t)}{\lambda w_{H}}+H_{Y}\left(w_{H}, L\right)=H \\
& a_{L Y}\left(w_{H}, w_{L}\right) Y\left(w_{H}, L\right)=L
\end{aligned}
$$

em que

$Y\left(w_{H}, L\right)$ : oferta do bem homogêneo, $Y$;

$H_{Y}\left(\mathfrak{w}_{H}, L\right)$ : procura de capital humano para a produção do bem $Y$, quando emprega a totalidade da mão-de-obra não-qualificada. 
Por outro lado, da igualdade Despesa $=$ Rendimento podemos retirar a seguinte condição de equilíbrio no mercado dos bens (diferenciados e homogêneo):

$$
E(t)+Y(t)=w_{L} L+w_{H} H+\pi(t)-a_{H I} w_{H} i(t)
$$

o que, conjugado com a condição (8) de maximização da utilidade instantânea, $\frac{\alpha}{1-\alpha}=\frac{E(t)}{Y(t)}$, e a expressão (12) do fluxo de lucro do monopolista $\pi(t)=\left(1-\frac{1}{\lambda}\right) E(\mathrm{t})$, origina

$$
E(t)=\frac{w_{L} L+w_{H} H-a_{H I} w_{H} i(t)}{\left(\frac{1}{\alpha}+\frac{1}{\lambda}-1\right)}
$$

ou seja, a condição (simplificada) do equilíbrio no mercado dos bens (diferenciados e homogêneo).

\subsection{Taxa de Inovação de Equilibrio de Mercado}

Considerando as equações que descrevem a condição de otimização do consumo (9), a expressão do lucro (12), e a condição de não-arbitragem (14), resolvemos o sistema de modo a encontrar a intensidade do esforço de pesquisa (isto é, a taxa de inovação) de equilíbrio. Assim, substituindo (9), expressão da trajetória temporal ótima dos gastos $\frac{\dot{E}}{E}=r-\rho$, e (12), fluxo de lucro do monopolista $\pi(t)=\left(1-\frac{1}{\lambda}\right) E(\mathrm{t})$, na condição de não-arbitragem (14), $\frac{\pi+\dot{v}}{v}-i=r$, obtém-se:

$$
\frac{\dot{v}}{v}=\rho+i+\frac{\dot{E}}{E}-\frac{\left(1-\frac{1}{\lambda}\right) E}{v}
$$

No equilíbrio de steady-state, $\frac{\dot{v}}{v}=\frac{\dot{E}}{E}=0$, o que, substituído em (18) e (9), implica, respectivamente:

$$
\left(1-\frac{1}{\lambda}\right) E=(\rho+i) v
$$




$$
r=\rho
$$

Da equação (19) obtém-se que $E=\frac{(\rho+i) v}{\left(1-\frac{1}{\lambda}\right)}$, o que, conjugando com a condição de livre-entrada (13), $a_{H I} w_{H}=p$, origina $E=\frac{(\rho+i) a_{H I} w_{H}}{\left(1-\frac{1}{\lambda}\right)}$. Combinando esta última equação com a condição de equilíbrio do mercado dos bens (17), $E(t)=\frac{w_{L} L+w_{H} H-a_{H I} w_{H} i(t)}{\left(\frac{1}{\alpha}+\frac{1}{\lambda}-1\right)}$, obtemos, após alguma manipulação algébrica, a taxa de inovação de equilíbrio de mercado:

$$
i^{e}=\frac{\alpha\left(1-\frac{1}{\lambda}\right)\left(\frac{w_{L}}{w_{H}} L+H\right)}{a_{H I}}-\frac{\rho}{\lambda}[\lambda-\alpha(\lambda-1)]
$$

Dada a natureza específica da utilização do fator trabalho não-qualificado, esta taxa de inovação de equilíbrio é a taxa que resulta da escolha descentralizada, pelos empresários, de como (da forma mais lucrativa) afetar o capital humano que não é usado na produção do bem homogêneo para a produção de produtos diferenciados versus produção de inovações (atividade de pesquisa).

Como detalharemos mais à frente na subseção 2.7 , a taxa de inovação de equilíbrio de mercado é tanto maior quanto maior for a dotação da economia em capital humano $(H)$; quanto menor for a dotação da economia em mão-de-obra não-qualificada $(L)$, admitindo elasticidade de substituição na produção do bem homogêneo pouco elevada; quanto maior for a produtividade $\left(1 / a_{H I}\right)$ da pesquisa; quanto mais pacientes forem as famílias (menor for a taxa de preferência intertemporal; quanto maior for a dimensão dos avanços tecnológicos (dos "degraus" de qualidade); e quanto maior for o grau de "sofisticação" dos consumidores $(\alpha)$. 


\subsection{Taxa de Crescimento do Índice de Consumo}

Considerando as equações referentes à utilidade instantânea do consumo agregado de $X(2)$, as funções-procura estática para os produtos diferenciados (6), o equilíbrio do mercado dos bens (8) e a fixação do preço-limite (10), obtém-se (note-se que a expressão do lado esquerdo é o logaritmo do índice de consumo):

$$
\begin{aligned}
\alpha \log u(t)+(1-\alpha) \log y(t) & =\alpha \int_{0}^{1}\left(\log \sum_{j} q_{j}(k) d k\right)+\log E(t)-\alpha \log \lambda-\alpha \log w_{H}+ \\
& +(1-\alpha) \log \left(\frac{1-\alpha}{\alpha}\right)
\end{aligned}
$$

Consideremos agora:

$f(j, t)$ : a probabilidade de, para um produto qualquer $k$, existirem $j$ melhorias na qualidade, durante o intervalo de tempo $t$.

Como, em equilíbrio, todos os produtos têm a mesma intensidade de pesquisa, $f(j, t)$ representa a fração dos produtos que são melhorados $j$ vezes durante o intervalo $t$. Ou seja, a contabilização de todos os produtos e todas as gerações possíveis gera (Grossman e Helpman, 1991b):

$$
\int_{0}^{1} \log q_{t}(k) d k=\sum_{j=0}^{\infty} f(j, t) \log \lambda^{j}
$$

Como $\sum_{j=0}^{\infty} f(j, t) \log \lambda^{j}=\sum_{j=0}^{\infty} f(j, t) j \log \lambda=\mathbf{E}(j)$, pelas propriedades da distribuição de Poisson resulta: ${ }^{16}$

$$
\int_{0}^{1} \log q_{t}(k) d k=i t \log \lambda
$$

Substituindo (24) em (22) temos:

$\alpha \log u(t)+(1-\alpha) \log y(t)=\alpha i \log \lambda+\log E-\alpha \log w_{H}+(1-\alpha) \log \left(\frac{1-\alpha}{\alpha}\right)-\alpha \log \lambda$

$16 f(j, t)=p(j)=\frac{(t)^{j} e^{-t}}{j !}$ e $\mathbf{E}(\mathrm{j})=i t$, pelas propriedades da distribuição de Poisson, em que $E$ representa aqui o valor esperado (ver Santos, 1988, p. 245). 
Assim, a taxa de crescimento do índice de consumo é (note-se que em equilíbrio $E$ e $i$ são constantes, enquanto $\mathfrak{w}_{H}$ é determinado globalmente pelas dotações de fatores que consideramos fixas):

$$
g \equiv \frac{d \log \left[u(t)^{\alpha} y(t)^{1-\alpha}\right]}{d t}=\alpha i \log \lambda
$$

\subsection{Estática Comparada do Equilibrio de Mercado}

Recorde-se que $i$ é, em (21), dado por $i^{e}=\frac{\alpha\left(1-\frac{1}{\lambda}\right)\left(\frac{w_{L}}{w_{H}} L+H\right)}{a_{H I}}-\frac{\rho}{\lambda}[\lambda-\alpha(\lambda-1)]$. Assim:

$$
g=\alpha \log \lambda\left\{\frac{\alpha\left(1-\frac{1}{\lambda}\right)\left(\frac{w_{L}}{w_{H}} L+H\right)}{a_{H I}}-\frac{\rho}{\lambda}[\lambda-\alpha(\lambda-1)]\right\}
$$

Da análise de (26), constatamos que a taxa de crescimento da economia ( $g \equiv$ taxa de crescimento do índice do consumo) é tanto maior quanto,

a) mais rápida for a taxa de inovação, $\frac{\partial g}{\partial i}=\alpha \log \lambda>0$.

b) maior for $\alpha$, o peso dos produtos diferenciados no índice de consumo,

$$
\frac{\partial g}{\partial \alpha}=i \log \lambda+\frac{\partial g}{\partial i} \cdot \frac{\partial i}{\partial \alpha}=i \log \lambda+\alpha \log \lambda\left[\frac{\left(1-\frac{1}{\lambda}\right)\left(\frac{w_{L}}{w_{H}} L+H\right)}{a_{H I}}+\frac{\rho}{\lambda}(\lambda-1)\right]>0
$$

Ou seja, quanto mais sofisticados forem os consumidores, no sentido de terem uma maior preferência relativa pelos produtos diferenciados de melhor qualidade (isto é, de serem mais sensíveis à qualidade), maior é a taxa de crescimento da economia. ${ }^{17}$

17 Seria interessante considerar a hipótese de que a maior sofisticação dos consumidores está positivamente correlacionada com o nível de capital humano. Neste caso, que não analisamos aqui explicitamente, o capital humano influenciaria também a procura, pois $\alpha$ seria função positiva de $H$. Estaríamos na presença de um canal adicional de influência do capital humano sobre o crescimento econômico. 
Da análise de (27) conclui-se, adicionalmente, que a taxa de inovação (e, logo, a taxa de crescimento) é tanto maior quanto,

c) maior for a dotação da economia em capital humano

$$
\frac{\partial i}{\partial H}=\frac{\alpha\left(1-\frac{1}{\lambda}\right)}{a_{H I}}\left[1+\frac{\left(\frac{\partial w_{L}}{\partial H} w_{H}-\frac{\partial w_{H}}{\partial H} w_{L}\right) L}{\left(w_{H}\right)^{2}}\right]>0 \quad \text { pois } \quad \frac{\partial w_{L}}{\partial H}>0 \text { e } \frac{\partial w_{H}}{\partial H}<0
$$

As remunerações dos fatores são fixas para cada nível de dotação de fatores. Assim, um aumento da quantidade de capital humano disponível origina uma relativa escassez de $L$, levando a um aumento na remuneração deste fator. Por sua vez, dada a relativa abundância de capital humano, a remuneração deste fator tende a diminuir, baixando os custos da atividade de pesquisa e aumentando o ritmo de crescimento.

d) menor for a dotação da economia em mão-de-obra não-qualificada, admitindo elasticidade de substituição na produção do bem homogêneo pouco elevada

$$
\begin{array}{r}
\frac{\partial i}{\partial L}=\frac{\alpha\left(1-\frac{1}{\lambda}\right)}{a_{H I}}\left[\frac{w_{L}}{w_{H}}+\frac{\left(\frac{\partial w_{L}}{\partial L} w_{H}-\frac{\partial w_{H}}{\partial L} w_{L}\right) L}{\left(w_{H}\right)^{2}}\right]<0 \quad \text { pois } \quad \frac{\partial w_{L}}{\partial L}<0 \text { e } \frac{\partial w_{H}}{\partial L}>0 \\
\text { e admitindo que }\left|\frac{\partial w_{L}}{\partial L}-\frac{\partial w_{H}}{\partial L} \frac{w_{L}}{w_{H}}\right|>\frac{w_{L}}{L}
\end{array}
$$

Este resultado deriva do fato de o bem homogêneo, $Y$, utilizar na sua produção capital humano $(H)$ e trabalho não-qualificado $(L)$. Ora, um aumento no fator específico a este setor - o fator $L$ - diminui a respectiva remuneração e aumenta a remuneração do capital humano, por se tornar relativamente mais escasso. Assumindo que o efeito do aumento da remuneração relativa de $H$ é maior que o efeito de substituição de $H$ por $L$ na produção de $Y$, ${ }^{18}$ a taxa de inovação decai, assim como a taxa de crescimento da economia.

e) maior a produtividade $\left(\mathrm{l} / a_{H I}\right)$ da pesquisa, $\frac{\partial i}{\partial a_{H I}}=-\frac{\alpha\left(1-\frac{1}{\lambda}\right)\left(\frac{w_{L}}{w_{H}} L+H\right)}{\left(a_{H I}\right)^{2}}<0$.

18 Globalmente, o aumento de $L$ torna o capital humano relativamente mais escasso. Mas, simultaneamente, liberta parte do capital humano para a produção de bens diferenciados e investigação. $\mathrm{O}$ que admitimos aqui é que a elasticidade de substituição na produção de $Y$ não é suficientemente elevada para tornar o segundo efeito dominante. 
Um aumento da produtividade incrementa o retorno esperado da atividade de pesquisa, incentivando, assim, os esforços/investimentos na P\&D, e aumenta, conseqüentemente, o crescimento via aumento da taxa de inovação.

f) mais pacientes forem as famílias (menor for a taxa de preferência intertemporal, $\rho$ ) $\frac{\partial i}{\partial \rho}=-\frac{1}{\lambda}[\lambda-\alpha(\lambda-1)]<0$.

Um comportamento, por parte das famílias, mais propenso à poupança, faz diminuir o custo de financiamento, o que contribui para aumentar a taxa de inovação e, conseqüentemente, a taxa de crescimento.

Finalmente, obtemos também de (25) e (26) o efeito de um aumento na dimensão dos avanços tecnológicos de qualidade $(\lambda)$. O crescimento é tanto maior quanto,

g) maior for a dimensão dos avanços tecnológicos (dos "degraus" de qualidade) $\frac{\partial g}{\partial \lambda}=\frac{\alpha i}{\lambda}+\alpha \log \lambda \frac{\partial i}{\partial \lambda}=\frac{\alpha i}{\lambda}+\frac{\alpha^{2} \log \lambda}{\lambda^{2}}\left[\frac{\left(\frac{w_{L}}{w_{H}} L+H\right)}{a_{H I}}+\rho\right]>0$.

Note-se que o aumento na dimensão dos avanços da qualidade $(\lambda)$ promove o crescimento por duas vias: diretamente, pois os degraus de qualidade são maiores; indiretamente, originando uma mais rápida ocorrência de avanços tecnológicos, aumentando, portanto, o retorno esperado da pesquisa, o que contribui para atrair recursos (capital humano) para as atividades de P\&D.

h) maior for o grau de "sofisticação" dos consumidores $(\alpha)$

$$
\frac{\partial i}{\partial \alpha}=\left[\frac{\left(1-\frac{1}{\lambda}\right)\left(\frac{w_{L}}{w_{H}} L+H\right)}{a_{H I}}+\frac{\rho}{\lambda}(\lambda-1)\right]>0 \text {. }
$$

Um corolário importante desta análise é a previsão (ceteris paribus) de uma taxa de crescimento menos elevada para as economias de maior dimensão (com maior quantidade de mão-de-obra não qualificada). Em contrapartida, a dimensão do capital humano é crucial, sendo que quanto mais elevada, mais rápido será o crescimento. 
Isto está em consonância com as conclusões de Romer (1990) “... that the correct measure of scale is not population but human capital...” (p. $S_{78}$ ) e que uma economia “... with a larger total stock of human capital will experience faster growth." (p. $\left.S_{99}\right)$.

Neste contexto, uma economia de grande dimensão, com abundância de trabalho qualificado $(H)$, realiza mais pesquisa industrial porque o setor de P\&D utiliza este fator mais intensivamente. Tal economia cresce mais rapidamente que outra, com características semelhantes, mas com uma menor quantidade de capital humano. No entanto, uma economia de grande dimensão, habitada, em grande parte, por indivíduos não-qualificados ( $L$ elevado), pode crescer mais lentamente que uma outra, idêntica, mas com uma menor população.

Em outras palavras, segundo conclusão de Grossman e Helpman (1994, p. 36): ${ }^{19}$ "[t] he larger labor-abundant country, which specializes relatively in labor-intensive production, might will conduct absolutely less industrial research than a smaller country with comparative advantage in $R \& D . "$

\section{ANÁLISE DE BEM-ESTAR}

\subsection{Considerações Iniciais}

Sob as hipóteses de tecnologia exógena e plena apropriabilidade do investimento, e assumindo que os indivíduos possuem antevisão de longo prazo perfeita (relativamente ao seu comportamento de poupança) e valorizam o bem-estar dos seus descendentes, no modelo neoclássico tradicional os incentivos de mercado geram um ritmo de crescimento econômico socialmente ótimo, não havendo lugar, conseqüentemente, para um papel ativo e interventor dos governos. Em outras palavras, no modelo neoclássico de Solow as trajetórias do crescimento de equilíbrio de mercado são também socialmente eficientes.

Mas em modelos em que o crescimento econômico é endogeneizado - ou, mais especificamente, conduzido por inovações endógenas (como é o caso do modelo aqui desenvolvido) -, as não-convexidades associadas fazem com que a taxa de crescimento de equilíbrio de mercado não coincida com a taxa de crescimento socialmente ótima (de Pareto). Por um lado, o ótimo de Pareto exige que o preço iguale o custo marginal da produção, o que está em desacordo com o modelo, dado que a inovação exige

19 Estas conclusões sugerem implicitamente, como referido na revisão da literatura, que o livre comércio internacional poderá contribuir para acelerar o crescimento. Para uma análise mais detalhada acerca da acumulação do capital humano e interligação deste com as questões do comércio internacional, ver Grossman e Helpman (1993, capítulo 5). 
a existência de lucros monopolistas. Por outro lado, o ótimo de Pareto exige que o retorno da acumulação de capital (investimento) seja completamente apropriado, o que não acontece na presença de spillovers de conhecimento. Cada inovador, analisando o produto em questão (na sua geração mais recente), constrói o seu próprio produto a partir dele, apropriando-se, assim, de uma fração do investimento acumulado em conhecimento que nele está incluído.

Nesse quadro, a comparação entre a taxa de inovação endógena e a taxa de inovação socialmente desejável é, de início, ambígua. A existência de spillovers intertemporais e a incapacidade dos inovadores para captarem todo o retorno dos esforços de pesquisa apontam para a insuficiência de incentivos de mercado para o investimento em conhecimento, ou seja, $i^{e}<i^{*}$, designando $i^{*}$ a taxa de inovação ótima de Pareto.

No entanto, pode também ocorrer investimento excessivo em P\&D $\left(i^{\mathrm{e}}>i^{*}\right)$, na medida em que os inovadores reagem aos sinais de lucro privado, que divergem, eventualmente, das medidas de rentabilidade social.

O subitem a seguir tenta esclarecer tal ambigüidade, determinando formalmente em que circunstâncias ocorrem investimentos excessivos em P\&D e em que circunstâncias eles são insuficientes.

\subsection{Taxa de Inovação Ótima de Pareto}

Suponhamos, por conveniência técnica de resolução do modelo, a existência de um planejador social, a quem cabe a resolução de duas questões: a alocação estática e a alocação dinâmica dos recursos existentes na economia, de forma a maximizar a utilidade social.

A utilidade total (1), vale lembrar, é dada por:

$$
\mathrm{U}=\int_{0}^{\infty} \mathrm{e}^{-\rho \mathrm{t}} \mathrm{C}(\mathrm{t}) \mathrm{dt}=\int_{0}^{\infty} \mathrm{e}^{-\rho \mathrm{t}}[\alpha \log \mathrm{u}(\mathrm{t})+(1-\alpha) \log \mathrm{Y}(\mathrm{t})] \mathrm{dt} .
$$

\section{Alocação Estática}

A cada momento, o planejador social, tendo em conta o capital humano existente e tomando a despesa total $(E+Y)$ como dada, decide qual deve ser a distribuição deste capital entre as atividades de pesquisa e de produção dos bens diferenciados e do bem homogêneo. ${ }^{20}$

20 A alocação do outro recurso - o trabalho não-qualificado - é trivial, pois $L$ é específico da produção de $Y$. 
Por $(2), \alpha \log u(t)+(1-\alpha) \log Y(t)=\alpha \int_{0}^{1} \log \left(\sum_{j} q_{j}(k) d_{j t}(k)\right) d k+(1-\alpha) \log Y(t)$.

Como explicado nos subitens 2.2 e 2.4 , as preferências do consumidor representativo implicam que o emprego de capital humano na produção dos bens diferenciados seja $a_{H X} \frac{E}{\lambda c_{X}}=a_{H X} \frac{E}{\lambda a_{H X} w_{H}}$, e na produção do bem homogêneo $a_{H Y} \frac{1-\alpha}{\alpha} E$.

O emprego de trabalho não-qualificado é $a_{L Y} \frac{1-\alpha}{\alpha} E$. Sendo assim, as restrições sobre os recursos são as seguintes:

$$
\begin{aligned}
& \int_{0}^{1}\left(\sum_{j} d_{j}(k)\right) d k \leq \frac{E}{\lambda w_{H}} \\
& Y \leq \frac{1-\alpha}{\alpha} E
\end{aligned}
$$

Como todas as qualidades de qualquer produto exigem o mesmo input de capital humano, o planejador produz apenas a qualidade estado-de-arte, $q_{t}$. Assim:

$$
\alpha \log u(t)+(1-\alpha) \log Y(t)=\alpha \int_{0}^{1} \log q_{t}(k) d k+\alpha \int_{0}^{1} \log d_{t}(k) d k+(1-\alpha) \log Y(t)
$$

A maximização do índice de consumo - ou do seu logaritmo, (30) -, sujeito às restrições (28) e (29) resulta numa distribuição idêntica de capital humano para todas as indústrias dos bens diferenciados e numa distribuição entre bens diferenciados, por um lado, e o bem homogêneo, por outro, proporcional aos respectivos pesos nas preferências dos consumidores. Assim, a alocação ótima de recursos, para cada indústria, em cada momento do tempo, é dada por:

$$
\begin{array}{ll}
\mathrm{d}_{\mathrm{t}}(\mathrm{k})=\frac{\mathrm{E}(\mathrm{t})}{\lambda \mathrm{w}_{\mathrm{H}}} & \forall \mathrm{k} \in[0,1] \\
Y(t)=\frac{1-\alpha}{\alpha} E(t) &
\end{array}
$$

Por outro lado, da condição de equilíbrio do mercado dos bens (homogêneo e diferenciados) tem-se, tal como se constatou no subitem 2.4, 


$$
E(t)+Y(t)=w_{L} L+w_{H} H+\pi(t)-a_{H I} w_{H} i(t)
$$

Utilizando (12) e (32) podemos simplificar a expressão (33), obtendo

$$
\left(\frac{1}{\alpha}+\frac{1}{\lambda}-1\right) E(t)=w_{L} L+w_{H} H-a_{H I} w_{H} i(t)
$$

\section{Alocação Dinâmica}

Trata-se agora de, dada a alocação estática para cada nível de despesa $(E+Y)$, escolher uma seqüência ótima de despesa que maximize a utilidade total intertemporal.

Utilizando (31) e (32) em (30), a expressão da utilidade total será:

$U_{t}=\int_{0}^{\infty} e^{-\alpha t} C(t) d t=\int_{0}^{\infty} e^{-\alpha}\left[\alpha \int_{0}^{1} \log q_{t}(k) d k+\alpha \log \left(\frac{E}{\lambda w_{H}}\right)+(1-\alpha) \log \left(\frac{1-\alpha}{\alpha}\right) E\right] d t$

Quer dizer, a maximização de $U_{t}$ exige que se considerem as expressões de $\log u(t)$ e $\log Y(t)$ derivadas da alocação ótima de recursos para cada indústria, em cada momento do tempo.

Quanto à produção de melhorias de qualidade, vale relembrar que:

$\int_{0}^{1} \log q_{t}(k) d k=\log \lambda \times\left(n^{o}\right.$ de melhorias que ocorrem na indústria até ao momento $\left.t\right)$.

As melhorias ocorrem em cada indústria de acordo com um processo de Poisson variante no tempo, com uma taxa de ocorrência instantânea $i(s)$. Assim:

$\mathrm{N}^{\mathrm{o}}$ de sucessos esperados antes do momento $\mathrm{t}=I(t)=\int_{0}^{t} i(s) d s$

Substituindo em (35), $U_{t}=\int_{0}^{\infty} e^{-\rho t}\left[(\alpha \log \lambda) I(t)+\alpha \log \left(\frac{E(t)}{\lambda w_{H}}\right)+(1-\alpha) \log \left(\frac{1-\alpha}{\alpha}\right) E(t)\right] d t$,

e rearranjando, tem-se:

$U_{t}=\int_{0}^{\infty} e^{-\rho t}\left[(\alpha \log \lambda)(I(t)-1)+\log E-\alpha \log w_{H}+(1-\alpha) \log \left(\frac{1-\alpha}{\alpha}\right)\right] d t$ 


\section{Objetivo do Planejador Social}

Dada a condição de equilíbrio do mercado dos bens, (34), o planejador social escolhe uma sequiência de $E(t)$ (variável de controle ${ }^{21}$ de modo a maximizar a função objetivo (utilidade total) sujeita a determinadas restrições. Estas restrições são dinâmicas, dado que descrevem a evolução da economia ao longo do tempo, tal como é representada pelo conjunto das variáveis estado $\left(H, L, w_{L}, w_{H}\right.$ e $\left.I\right) .^{22}$

Em termos formais:

$$
\begin{aligned}
& \underset{\{E(t)\}}{\operatorname{Max}} U_{t}=\int_{0}^{\infty} e^{-\rho t}\left[(\alpha \log \lambda)(I(t)-1)+\log E(t)-\alpha \log w_{H}+(1-\alpha) \log \left(\frac{1-\alpha}{\alpha}\right)\right] d t \\
& \left.\begin{array}{l}
\text { S.a }(t)=i(t) \\
\left(\frac{1}{\alpha}+\frac{1}{\lambda}-1\right) E(t)=w_{L} L+w_{H} H-a_{H I} w_{H} i(t) \quad \text { (II. 34) }
\end{array}\right\} \dot{I}(t)=\left(\frac{\frac{w_{L}}{w_{H}} L+H-\left(\frac{1}{\alpha}+\frac{1}{\lambda}-1\right) \frac{E(t)}{w_{H}}}{a_{H I}}\right) \\
& I(t)>0
\end{aligned}
$$

Para resolver este problema dinâmico, utiliza-se o Princípio do Máximo do Controle Ótimo (Pontryagin, Boltyanskii, Gamkrelidze e Mishchenko, 1962). A construção da Função Hamiltoniana (em valor corrente) constitui o ponto de partida para a resolução do problema:

$\operatorname{Hcv}\left(E, \alpha, \lambda, I, w_{H}, w_{L}, \theta, t\right)=(\alpha \log \lambda)(I(t)-1)+\log E(t)-\alpha \log \lambda-\alpha \log w_{H}+$

$$
+(1-\alpha) \log \left(\frac{1-\alpha}{\alpha}\right)+\theta\left(\frac{\frac{w_{L}}{w_{H}} L+H}{a_{H I}}-\left(\frac{1}{\alpha}+\frac{1}{\lambda}-1\right) \frac{E(t)}{w_{H} a_{H I}}\right)
$$

$21 \mathrm{Na}$ terminologia do controle ótimo, os fluxos denominados de variáveis de controle e os estoques" de variáveis estado. (Dixit, 1990).

22 Note-se que na resolução do problema de controle ótimo acabamos por ter somente uma variável estado, $I$, pois as outras são exógenas $\left(H, L\right.$, diretamente e $w_{L}$ e $w_{H}$ devido à exogeneidade das primeiras e da especificidade de $L$ ). 
em que $\theta$ (variável co-estado) é o preço-sombra da intensidade do esforço de pesquisa.

Segundo o Princípio de Prontryagin, as condiçôes necessárias para a solução ótima do problema do planejador social são as seguintes:
i) $\underset{\{E(t)\}}{\operatorname{Max}} \mathrm{Hcv}$;
ii) $\dot{I}(t)=\frac{\frac{w_{L}}{w_{H}} L+H}{a_{H I}}-\left(\frac{1}{\alpha}+\frac{1}{\lambda}-1\right) \frac{E(t)}{a_{H I} w_{H}}$
iii) $\dot{\theta}(t)=\rho \theta(t)-\frac{\partial H c v}{\partial I}$;
iv) $\lim _{t \rightarrow \infty} \theta(t) I(t) e^{-\rho t}=0$

A condição $i$ ), supondo uma solução interior, resulta em:

$$
\text { i) } \underset{\{E(t)\}}{\operatorname{Max}} H c v \Rightarrow \frac{d H c v}{d E(t)}=0 \Rightarrow E=\frac{w_{H} a_{H I}}{\theta\left(\frac{1}{\alpha}+\frac{1}{\lambda}-1\right)}
$$

A condição ii), chamada de "equação de movimento", obriga a solução a obedecer à restrição do problema. Quanto à chamada "equação de co-estado" - condição iii) -, resulta em:

$$
\frac{\dot{\theta}}{\theta}=\rho-\frac{\alpha \log \lambda}{\theta}
$$

Admitindo que as funções consideradas no princípio Hamiltoniano são côncavas em $E$ e $I$, estas condições necessárias são também suficientes. (Mangasarian, 1966). A partir delas podemos derivar a solução de crescimento equilibrado, para a qual o sistema converge a partir de uma situação inicial qualquer. Interessa agora definir as soluções de crescimento equilibrado para onde se espera que o sistema convirja. Neste equilíbrio de steady-state, a taxa de crescimento da despesa total em produtos diferenciados, a taxa de acumulação dos esforços de pesquisa e a taxa de crescimento do preço-sombra dos esforços de pesquisa são constantes (isto é, $\widehat{\boldsymbol{E}}, \hat{\boldsymbol{I}}, \hat{\theta}$ são constantes). Combinando a "equação de co-estado" (43) com a condição de transversalidade iv), obtém-se:

$$
\theta=\frac{\alpha \log \lambda}{\rho}
$$


Assim, de (42) resulta:

$$
E=\frac{\rho w_{H} a_{H I}}{\alpha \log \lambda\left(\frac{1}{\alpha}+\frac{1}{\lambda}-1\right)}
$$

Pela "equação de movimento", e utilizando (38) e (45), conclui-se que:

$$
i^{*}=\frac{\frac{w_{L}}{w_{H}} L+H}{a_{H I}}-\frac{\rho}{\alpha \log \lambda}
$$

A equação (46) representa a expressão da taxa de inovação ótima de Pareto.

Tal como no equilíbrio descentralizado (de mercado), a taxa de inovação ótima é tanto maior (ceteris paribus) quanto: a) maior a dotação da economia em capital humano; b) maior a porcentagem de produtos diferenciados no índice de consumo; c) menor a dotação da economia em mão-de-obra não-qualificada (admitindo elasticidade de substituição na produção do bem homogêneo pouco elevada); d) maior a produtividade do setor de P\&D; e) menor a taxa de substituição intertemporal; f) maiores os avanços na qualidade dos produtos.

\subsection{O Problema da Insuficiência/Excesso de Incentivos para a P\&D}

Da análise efetuada nos subitens 2.4 e 3.2 , respectivamente, retiramos que a taxa de

inovação de equilíbrio de mercado é $i^{e}=\frac{\alpha\left(1-\frac{1}{\lambda}\right)\left[\left(\frac{w_{L}}{w_{H}}\right) L+H\right]}{a_{H I}}-\rho\left[1-\alpha\left(1-\frac{1}{\lambda}\right)\right]$

e a taxa de inovação ótima de Pareto é $i^{*}=\frac{\left(\frac{w_{L}}{w_{H}}\right) L+H}{a_{H I}}-\frac{\rho}{\alpha \log \lambda}$.

A diferença entre as taxas de inovação ótima e a de equilíbrio de mercado vem a ser:

$$
i^{*}-i^{e}=\left[\frac{\left(\frac{w_{L}}{w_{H}}\right) L+H}{a_{H I}}+\rho\right] \cdot\left[1-\alpha\left(1-\frac{1}{\lambda}\right)\right]-\frac{\rho}{\alpha \log \lambda}
$$


Os modelos endógenos de crescimento, em particular o modelo derivado aqui, são consistentes com a idéia de que as políticas governamentais podem afetar o ritmo de desenvolvimento econômico já que as taxas de inovação (e, portanto, de crescimento) ótima e de equilíbrio descentralizado podem não coincindir. Assim, os efeitos-crescimento temporários das políticas governamentais, implícitos no modelo neoclássico tradicional (Solow, 1956), são, nos novos modelos de crescimento endógenos, transformados em efeitos-crescimento permanentes. (Teixeira, 1999). Assim, estes modelos enfatizam a idéia de persistentes diferenças dos rendimentos per capita entre países: as economias mais ricas podem manter e até mesmo aumentar o fosso relativamente a economias mais pobres. Entre os determinantes do crescimento e (div)convergência identificada na literatura (Di Liberto, 2005), e em certa medida evidenciado no modelo proposto aqui, o capital humano é certamente um dos mais importantes.

Se o mecanismo de crescimento envolver externalidades positivas, constata-se que a taxa de crescimento de equilíbrio descentralizado é menor que a taxa de crescimento ótima de Pareto. Isto conduz à hipótese de a atuação do governo, na atribuição de subsídios para a investigação ou para a acumulação de capital humano, poder permitir

que o ótimo social seja atingido. ${ }^{23}$ No entanto, nos modelos estocásticos de produção de inovação, como no caso do apresentado neste artigo, é possível que se esteja investindo demasiado em investigação em termos do bem-estar social derivado da existência de uma externalidade negativa, o "business stealing effect" (ver item 4 do Apêndice). ${ }^{24}$ Nestes modelos, a questão de qual dos efeitos externos é dominante e, por conseguinte, a questão da diferença entre as mencionadas taxas de crescimento, depende da dimensão da inovação, do grau de poder de monopólio (dimensão das inovações) e, no caso concreto do nosso modelo, do grau de sofisticação dos consumidores (quota dos bens diferenciados). No caso de o grau de sofisticação dos consumidores ser relativamente baixo, induzindo, numa situação de equilíbrio de mercado, taxas de inovação e crescimento aquém do que seria socialmente ótimo, e admitindo que a sofisticação dos consumidores pudesse estar relacionada com o respectivo capital humano, existiria espaço para a política econômica, principalmente no sentido de subsidiar a acumulação de capital humano. Estando esta questão da política econômica e questões associadas à convergência/divergência das nações em termos de crescimento econômico para além do âmbito necessariamente estrito da presente investigação, elas constituiriam, com certeza, uma via de investigação futura interessante (para um survey acerca destes temas, ver Di Liberto, 2005).

23 Mesmo em Lucas (1988, p. 35), apesar de o autor apontar não ser seu objetivo "...[to] offer political advice", a existência de incentivos à acumulação de capital humano é suscetível de conduzir a uma situação de ótimo social. Em trabalhos mais recentes o autor é mais claro em relação a este aspecto, referindo-se, por um lado, ao enorme potencial de políticas de incentivo à acumulação de capital humano em contextos de imperfeição nos mercados de fatores (Lucas, 1990), e utilizando, por outro, os exemplos dos chamados "milagres asiáticos", caracterizados pelos fortes incentivos públicos à acumulação de capital humano, “... to help in assessing economic policies that may affect growth rates in other countries." (Lucas, 1993, p. 252).

$24 \mathrm{O}$ sobreinvestimento em I\&D ocorre porque os inovadores respondem aos sinais de lucros privados, que poderão divergir das medidas de rentabilidade social. 
Retomando a derivação algébrica acerca das circunstâncias em que o ótimo social e de mercado em termos de taxa de inovação podem divergir, temos que, numa situação de equilíbrio descentralizado, os agentes são incentivados a investir em P\&D $\left(i^{\mathrm{e}}>0\right)$ apenas quando:

$$
\left[\frac{\left(\frac{w_{L}}{w_{H}}\right) L+H}{\rho a_{H I}}+1\right]>\frac{\lambda}{\alpha(\lambda-1)}
$$

Por outro lado, $i^{*}>i^{e}$ quando:

$$
\left[\frac{\left(\frac{w_{L}}{w_{H}}\right) L+H}{\rho a_{H I}}+1\right]>\frac{\lambda}{(\alpha \log \lambda)[\lambda-\alpha(\lambda-1)]}
$$

A partir da análise destas duas expressões pode-se identificar diversas situações conforme a dimensão das inovações $(\lambda)$ e o peso do consumo dos bens diferenciados $(\alpha)$ no índice de consumo.

No sentido de clarificar tais situações, é útil recorrer a ilustrações gráficas construídas a partir das referidas expressões. O estudo analítico das expressões do lado direito das equações (48) e (49) permitiu identificar dois casos. Na eventualidade do peso dos bens diferenciados no índice do consumo ser relativamente baixo $(\alpha \leq 0.5)$ - Gráfico 2 - a taxa de equilíbrio de mercado situa-se sempre aquém da que seria socialmente desejável (ótima), independentemente da dimensão dos avanços tecnológicos. Assim, como se pode constatar no Gráfico 2, para lá da dimensão mínima exigida pelos agentes econômicos para se envolverem em atividades de pesquisa $\left(\lambda_{0}\right)$, a equação (49) é verificada. 


\section{GRÁFICO 2 - CASO EM QUE $\alpha \leq 0.5$. DIMENSÃO DO PROGRESSO TECNOLÓGICO ( $(\lambda)$ E DEFASAGEM ENTRE A TAXA DE INOVAÇÃO DE EQUILÍBRIO E A TAXA DE INOVAÇÃO ÓTIMA}

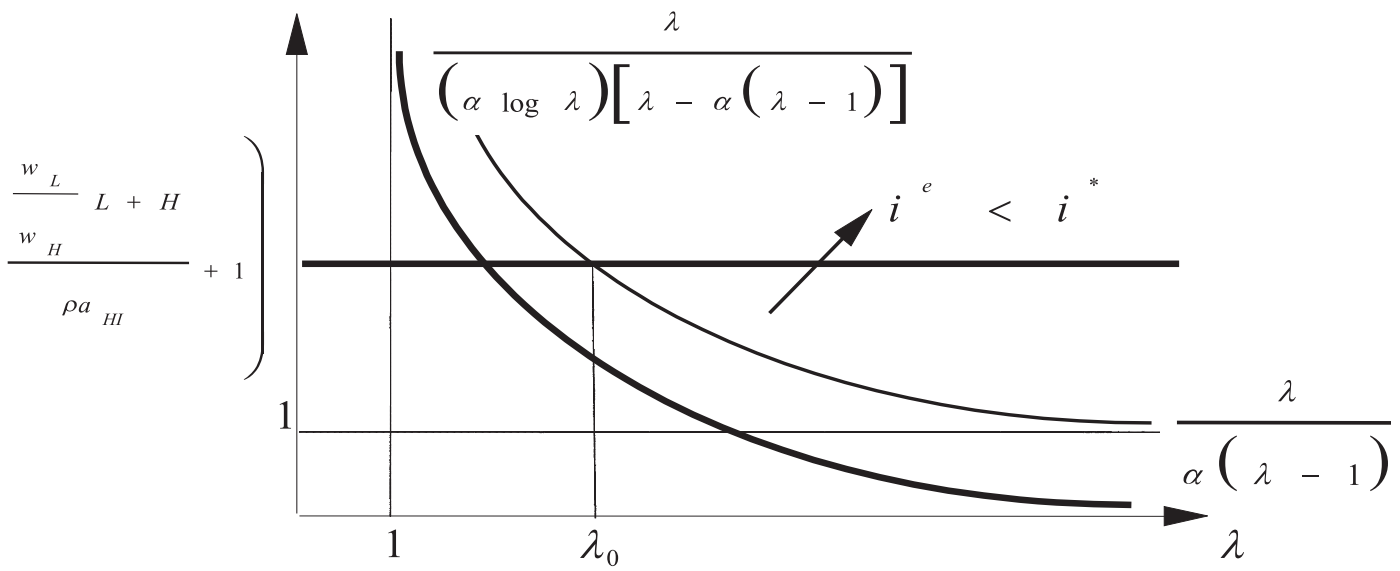

A não verificação de situações em que a taxa de inovação de mercado é excessiva relativamente ao bem-estar social explica-se pelo fato de, com baixa procura de bens diferenciados, este setor (que é onde a pesquisa pode gerar lucros) se tornar menos atrativo.

A ambigüidade ocorre quando os consumidores apresentam um consumo relativamente enviesado em direção aos bens diferenciados $(\alpha>0.5)$. Em face do mais elevado montante de lucros proporcionado por uma maior procura de bens diferenciados - como se verifica no caso ilustrado no Gráfico 3 -, existe a possibilidade de ocorrerem situações em que a taxa de equilíbrio de mercado gera incentivos excessivos em termos de bem-estar, ou seja, é superior à que seria socialmente adequada. Tais situações ocorrem no caso de o poder de monopólio (ou a dimensão do progresso tecnológico) ser relativamente reduzido, isto é, em termos formais, $\lambda \in] \lambda_{0}, \lambda_{1}[$. Para inovações de dimensões mais significativas, $\lambda \in] \lambda_{1},+\infty[$, a taxa de inovação do equilíbrio descentralizado revela-se socialmente insuficiente.

Os resultados obtidos neste ponto são distintos dos obtidos em modelos de inovação endógena análogos ao aqui desenvolvido, principalmente os de Grossman e Helpman (1991b, 1993), na base dos quais é feita a nossa análise. Tal diferença deriva fundamentalmente do fato de considerarmos um índice de consumo constituído por bens diferenciados e um bem homogêneo. Neste contexto, não é de estranhar que a constituição daquele índice (escolha por parte dos consumidores da alocação ótima da sua despesa entre os bens diferenciados e homogêneo) influencie a análise de bem-estar aqui efetuada. 


\section{GRÁFICO 3 - CASO EM QUE $\alpha>0.5$. DIMENSÃO DO PROGRESSO

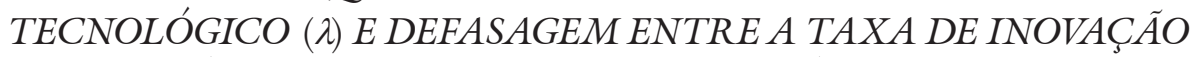 DE EQUILÍBRIO E A TAXA DE INOVAÇÃO ÓTIMA}

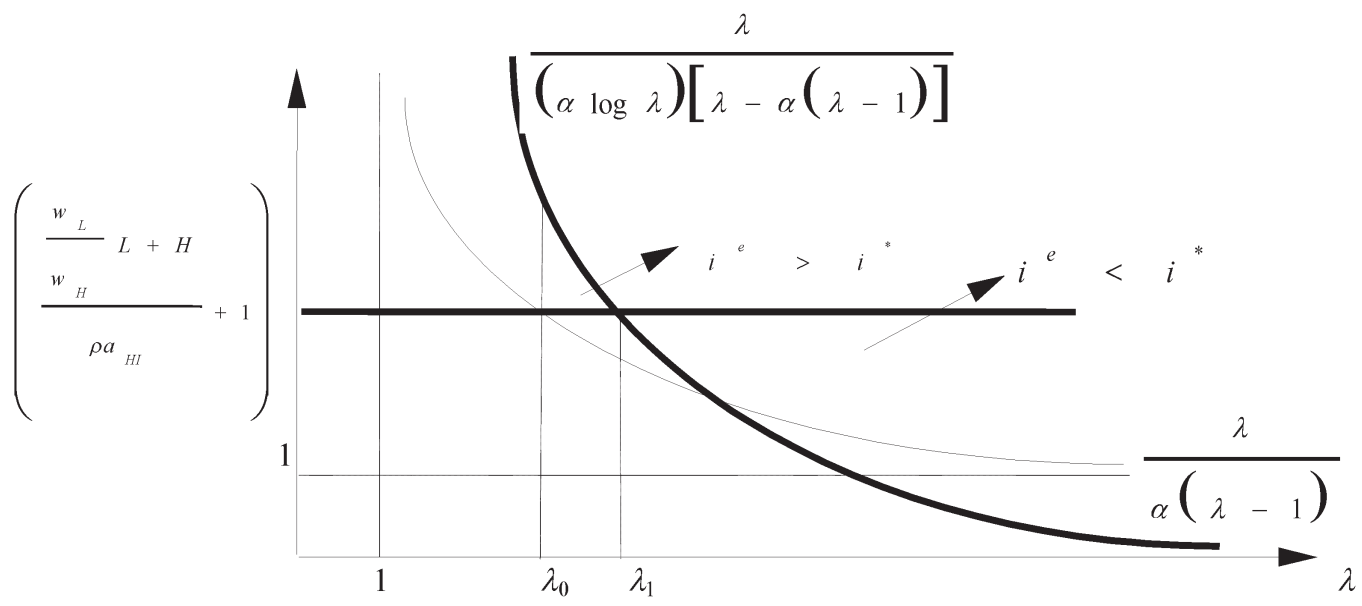

A procura tem, pois, uma influência determinante na insuficiência/excesso de incentivos ao investimento em pesquisa. Consumidores pouco sofisticados, isto é, com reduzida preferência por produtos diferenciados de qualidade, geram, seguramente, incentivos insuficientes para a pesquisa, enquanto que consumidores mais sofisticados podem gerar excesso de incentivos.

Se $\alpha$ (quota dos produtos diferenciados na procura) estiver positivamente correlacionada com o nível de desenvolvimento (no sentido lato) do país, então países menos desenvolvidos tendem a apresentar taxas de inovação relativamente reduzidas, ao contrário de países mais desenvolvidos, em que essas taxas podem, até, ser demasiado elevadas. ${ }^{25,} 26$

\section{CONCLUSÃO}

A emergência contínua de novas alternativas de consumo é uma característica sobejamente conhecida da dinâmica capitalista. No entanto, a compreensão do fenômeno

$25 \mathrm{O}$ fato de se estar considerando uma economia fechada pode ser aqui determinante. Como é comum em situações em que a dimensão da procura é relevante, a abertura ao exterior deve diminuir substancialmente a importância dessa dimensão.

26 A divergência entre a taxa de inovação de equilíbrio e a taxa de inovação ótima deriva da existência de não-convexidades, principalmente dos efeitos externos gerados por um inovador bem-sucedido, da fixação de preços não concorrenciais e dos efeitos de "criação destrutiva". No item 4 do Apêndice analisamos com mais detalhes esta divergência, identificando analiticamente os efeitos anteriormente mencionados, procurando, assim, interpretar economicamente os gráficos apresentados nesta seção. 
do lado da procura, associado à crescente relevância das atividades de consumo na sociedade, tem sido relativamente pouco explorada pela literatura do crescimento endógeno. Vários autores (e.g., Metcalfe, 2001; Dutt, 2006) chamam inclusive a atenção para o fato de as teorias neoclássica e endógena do crescimento econômico negligenciarem a procura agregada.

Tentando requalificar o papel-chave da procura no processo de crescimento econômico, o presente artigo derivou algebricamente um modelo de crescimento endógeno no qual o crescimento é induzido por melhorias na qualidade dos produtos.

À semelhança dos modelos de Grossman e Helpman (1991a, 1991b), cada inovação é construída a partir da anterior e cada nova geração do produto fornece proporcionalmente mais serviços que o produto da geração anterior (isto é, pressupomos, de forma razoável, que a dimensão da inovação, identificada no modelo pelo parâmetro $\lambda$, é sempre superior a 1 ).

De forma distinta daqueles autores, no entanto, consideramos um índice de consumo constituído por bens diferenciados e um bem homogêneo. Isto permite destacar a influência determinante da procura no crescimento econômico e atribuir aos consumidores a importância que estes realmente detêm na economia das nações.

Podemos sintetizar as previsões do modelo da seguinte forma:

* O crescimento da economia é tanto mais rápido quanto, ceteris paribus:

- maior for a dotação da economia em capital humano;

- menor for a dotação da economia em mão-de-obra não-qualificada (assumindo uma baixa elasticidade de substituição dos inputs na produção do bem homogêneo);

- maior for a produtividade da P\&D;

- mais pacientes forem os agentes econômicos;

- maior for a dimensão da inovação (dos “degraus” de melhoria dos produtos);

- maior for o peso dos bens diferenciados no índice de consumo.

* Existem falhas de mercado na alocação do capital humano aos setores que dele fazem uso. $\mathrm{O}$ investimento descentralizado em P\&D pode ser insuficiente ou excessivo em relação à alocação intertemporal ótima de recursos. 
No caso de um índice de consumo mais enviesado a favor do bem homogêneo, a taxa de inovação de mercado está sempre aquém do nível socialmente desejável, independentemente da dimensão das inovações. Esta última é importante na determinação da insuficiência/excesso de inovação no caso de os consumidores atribuírem maior peso aos bens diferenciados na constituição da sua cesta de consumo: no caso de inovações de reduzida dimensão, o investimento (descentralizado, de mercado) em inovação é socialmente excessivo (em termos do ótimo de Pareto).

Assim, o modelo proposto no presente artigo é consistente com a idéia de que as políticas governamentais podem afetar o ritmo de desenvolvimento econômico já que a taxa de inovação (e, portanto, de crescimento) ótima e de equilíbrio descentralizado pode não coincindir. Dependendo do efeito combinado do excedente do consumidor e dos spillovers intertemporais (externalidades positivas) estar além ou aquém do efeito destruição do lucro (externalidade negativa), a taxa de inovação (e crescimento) de equilíbrio descentralizado estará, respectivamente, aquém ou além do ótimo social. Isto depende crucialmente do grau de poder de monopólio (dimensão das inovações) e do grau de sofisticação dos consumidores (quota dos bens diferenciados).

No caso de o grau de sofisticação dos consumidores ser relativamente baixo, induzindo, numa situação de equilíbrio de mercado, a taxas de inovação e crescimento aquém do que seria socialmente ótimo, e admitindo-se, de forma razoável, que a sofisticação dos consumidores possa estar relacionada com o respectivo nível de capital humano, existiria espaço para a política econômica estar principalmente subsidiando a acumulação de capital humano.

Não obstante a questão da intervenção da política econômica e questóes de convergência/divergência dos países em termos de crescimento econômico não terem sido o enfoque do presente trabalho, certamente constituem uma via interessante a explorar em futuras investigações.

\section{REFEREANCIAS}

Aghion, P.; Howitt, P. A model of growth through creative destruction. Econometrica, v. 60, n. 2, p. 323-351, 1992.

Barro, R. Economic growth in a cross section of countries. Quarterly Journal of Economics, v. 106, n. 2, p. 407-443, 1991.

. Are government bonds net wealth? Journal of Political Economy, v. 81, n. 6, p. 1095-1117, 1974.

Barro, R. J.; Sala-i-Martin, X. Economic growth. New York: McGraw-Hill, Advanced Series in Economics, 1995. 
Becker, G. S.; Murphy, K. M.; Tamura, R. Human capital, fertility, and economic growth. Journal of Political Economy, v. 98, n. 5, p. S12-S37, 1990.

Bianchi, M. Novelty, preferences and fashion: when goods are unsettling. Journal of Economic Behavior and Organization, v. 47, p. 1-18, 2002.

Caballero, R. J.; Jaffe, A. B. How high are the giants shoulders: an empirical assessment of knowledge spillovers and creative destruction in a model of economic growth. National Bureau of Economic Research Macroeconomics Annual Meeting, p. 15-74, 1993.

Collins, S. M. Lessons from Korean economic growth. The American Economic Review, v. 80 , n. 2, p. 104-107, 1990.

Di Liberto, A. Convergence and divergence in neoclassical growth models with human capital. Contributi di Ricerca CRENoS (Centro Ricerche Nord-Sud, Università di Cagliari) n. 8, Cagliari, 2005.

Dixit, A. K. Optimization in economic theory. $2^{\text {nd }}$ Edition. New York: Oxford University Press, 1990.

Dutt, A. K. Aggregate demand, aggregate supply and economic growth. International Review of Applied Economics, v. 20, n. 3, p. 319-336, July 2006.

Fan, C. S. Quality, trade, and growth. Journal of Economic Behavior \& Organization, v. 55, p. 271-291, 2004.

Fatas-Villafranca, F; Saura-Bacaicoa, D. Understanding the demand-side of economic change: a contribution to formal evolutionary theorizing. Econ. Innow. New Techn., v. 13, n. 8, p. 695-716, 2004.

GEPIE. Inovação na indústria portuguesa: observatório MIE. Abril 1992.

Grossman, G. M.; Helpman, E. Endogenous innovation in the theory of growth. Journal of Economic Perspectives, v. 8, n. 1, p. 23-44, 1994.

. Innovation and growth in the global economy. Cambridge, Mass.; and London England: MIT Press, 2a impressão, 1993.

. Quality ladders and product cycles. Quarterly Journal of Economics, v. 106, n. 2 , p. 557-586, 1991 a.

. Quality ladders in the theory of growth. Review of Economic Studies, v. 58, p. 43-61, 1991b.

Helpman, E. Endogenous macroeconomic growth theory. European Economic Review, v. 36, p. $237-267,1992$.

Lucas, R. E. Making a miracle. Econometrica, v. 61, n. 2, p. 251-272, 1993.

. On the mechanics of economic development. Journal of Monetary Economics, v. 22 , p. 3-42, 1988.

Why doesn't capital flow from rich to poor countries? The American Economic Review, v. 80, n. 2, p. 92-96, 1990. 
Mangasarian, O. L. Sufficient conditions for the optimal control of nonlinear systems. SLAM Journal of Control, v. 4, p. 139-152, 1966.

Metcalfe, S. Consumption, preferences, and the evolutionary agenda. Journal of Evolutionary Economics, v. 11, p. 37-58, 2001.

Pasinetti, L. Structural change and economic growth. Cambridge: Cambridge University Press, 1981. 1993.

Pontryagin, L. S.; Boltyanskii, V. G.; Gamkrelidze, R. V.; Mishchenko, E. F. The mathematical theory of optimal processes. New York: Inter-science Publishers, 1962.

Ramsey, F. A mathematical theory of saving. Economic Journal, v. 38, p. 543-559, 1928; In: Stiglitz, J. E.; Uzawa, H. (eds.), Readings in the modern theory of economic growth. 5th. Ed. The MIT Press, 1979.

Romer, P. Growth based on increasing returns due to specialization. The American Economic Review, v. 77, p. 56-62, 1987.

. Endogenous technological change. Journal of Political Economy, v. 98, n. 5, pt.2, p. S71-S101, 1990.

Santos, F. B. Cálculo das probabilidades. 5a Edição. Plátano Editora SA, 1988.

Saviotti P. Information, variety and entropy in technoeconomic development. Research Policy, v. 17, p. 89-103, 1988. 1996.

. Technological evolution, variety and the economy. Aldershot: Edward Elgar,

. The role of variety in economic and technological development. In: Saviotti P.; Metcalfe, J.S. (eds.), Evolutionary theories of economic and technological change: present state and future prospects. Harwood Publishers, Reading, 1991, p. 172-208.

- Variety, economic and technological development. In: Shionoya Y.; Perlman, M. (eds.), Technology, industries and institutions: studies in Schumpeterian perspectives. Ann Arbor: The University of Michigan Press, 1994.

. Variety, growth and demand. Journal of Evolutionary Economics, v. 11, p. 119-142, 2001.

Saviotti P.; Mani G. S. Competition, variety and technological evolution: a replicator dynamics model. Journal of Evolutionary Economics, v. 5, p. 369-392, 1995.

Schultz, T. Investment in human capital. The American Economic Review, v. 51, n. 1, p. 1-17, 1961.

Schumpeter, J. A. The theory of economic development. Cambridge MA: Harvard University Press, 1934.

Segerstrom, P. S. Innovation, imitation, and economic growth. Journal of Political Economy, v. 99, n. 4, p. 807-827, 1991. 
Solow, R. M. A contribution to the theory of economic growth. Quarterly Journal of Economics, v. 70, p. 65-94, 1956.

Sonobe T.; Hu, D.; Otsuka, K. From inferior to superior products: an inquiry into the Wenzhou model of industrial development in China. Journal of Comparative Economics, v. 32, p. 542-563, 2004.

Teixeira, A. A. C. Capital humano e capacidade de inovação. Contributos para o estudo do crescimento Económico Português, 1960-1991. Série Estudos e Documentos, Conselho Económico e Social, Lisboa, 1999.

Witt, U. Economic growth - What happens on the demand side? Introduction. Journal of Evolutionary Economics, v. 11, p. 1-5, 2001a.

. Learning to consume - A theory of wants and the growth of demand. Journal of Evolutionary Economics, v. 11, p. 23-36, 2001 b. 


\section{APÊNDICE}

\section{RESOLUÇÃO DO PROBLEMA ESTÁTICO DO CONSUMIDOR}

Trata-se, aqui, de determinar a distribuição ótima da despesa total $(E+Y)$ para o consumidor representativo, em cada momento do tempo, entre o conjunto dos bens diferenciados $(E)$ e o bem homogêneo $(Y)$.

A utilidade instantânea global, em cada momento do tempo, é dada por:

$$
\alpha \log u(t)+(1-\alpha) \log y(t)=\alpha \log E(t)+\alpha \int_{0}^{1}\left(\log \frac{q_{t}(k)}{p_{t}(k)}\right) d k+(1-\alpha) \log Y(t)
$$

A restrição orçamentária, por sua vez, é dada por:

$$
E(t)+Y(t) \leq A(0)
$$

A resolução analítica do problema estático, ou seja, da maximização de (Al), sujeita a (A2), é imediata utilizando a função Lagrangiana:

$L(E, Y, \lambda)=\alpha \log E(t)+\alpha \int_{0}^{1}\left(\log q_{t}(k)-\log p_{t}(k)\right) d k+(1-\alpha) \log Y(t)+\lambda[A(0)-E(t)-Y(t)]$ em que:

$\lambda$ : multiplicador de Lagrange (preço sombra da restrição).

Admitindo que as funções incluídas no Lagrangiano são côncavas em $Y$ e $E$, as condições necessárias (e suficientes) para o ótimo são:
i) $\frac{\partial L}{\partial E}=0$
ii) $\frac{\partial L}{\partial Y}=0$
iii) $E+Y=A(0)$

Da resolução de i) e ii) resulta:
i) $\frac{\partial L}{\partial E}=0 \Rightarrow \lambda=\frac{\alpha}{E}$
$\Rightarrow Y(t)=\frac{1-\alpha}{\alpha} E(t)$
ii) $\frac{\partial L}{\partial Y}=0 \Rightarrow \lambda=\frac{1-\alpha}{Y}$ 
A expressão (A3) representa, assim, a relação entre a despesa no conjunto dos bens diferenciados $(E)$ e a despesa no bem homogêneo $(Y)$ que, em cada momento do tempo, maximiza a utilidade do consumidor representativo. Corresponde, portanto, à equação (8), no texto.

\section{RESOLUÇÃO DO PROBLEMA DINAMMICO DO CONSUMIDOR}

Problema do consumidor representativo:

$\underset{\{E(t), Y(t)\}}{\operatorname{Max}} U_{t}=\int_{0}^{\infty} e^{-\alpha}[\alpha \log u(t)+(1-\alpha) \log Y(t)] d t \quad$ s.a $\int_{0}^{\infty} e^{-R(t)}[E(t)+Y(t)] \leq A(0)$

Da resolução do problema estático retiramos que:

$\log u(t)=\log E(t)+\int_{0}^{1}\left(\log q_{t}(k)-\log p_{t}(k)\right) d k \quad e \quad Y(t)=\frac{1-\alpha}{\alpha} E(t)$

Desta forma, a utilidade total e a restrição orçamentária podem ser expressas como se segue:

$$
\begin{aligned}
U_{t}= & \int_{0}^{\infty} e^{-\rho t}\left[\log E(t)+\alpha \int_{0}^{1}\left(\log q_{t}(k)-\log p_{t}(k)\right) d k+(1-\alpha) \log \left(\frac{1-\alpha}{\alpha}\right)\right] d t \\
& \text { s.a } \\
& \frac{1}{\alpha} \int_{0}^{\infty} e^{-R(t)} E(t) d t \leq A(0)
\end{aligned}
$$

Uma forma simples de resolver o problema de optimização dado em (A6) passa pela utilização do Teorema de Kuhn-Tucker (1951). As condições de Kuhn-Tucker podem ser expressas a partir da função Lagrangiana:

$$
\begin{aligned}
L(E, t, \lambda) & =\int_{0}^{\infty} e^{-\alpha t}\left[\log E(t)+\alpha \int_{0}^{1}\left(\log q_{t}(k)-\log p_{t}(k)\right) d k+(1-\alpha) \log \left(\frac{1-\alpha}{\alpha}\right)\right] d t+ \\
& +\lambda\left[A(0)-\frac{1}{\alpha} \int_{0}^{\infty} e^{-R(t)}[E(t)] d t\right]
\end{aligned}
$$

em que: 
$\lambda$ : multiplicador de Lagrange, ou, em termos econômicos, o preço-sombra da restrição - utilidade extra que o agente obtém quando a restrição é relaxada em uma unidade.

Condições de $1^{a}$ Ordem para o Máximo
i) $\frac{\partial L}{\partial E}=0$
ii) $\frac{1}{\alpha} \int_{0}^{\infty} e^{-R(t)}[E(t)] \leq A(0), \lambda \geq 0$
iii) $\lambda\left[A(0)-\frac{1}{\alpha} \int_{0}^{\infty} e^{-R(t)}[E(t)] d t\right]=0$

A condição i) estabelece que uma condição necessária para que $E$ seja um máximo do problema com restrição é $E$ que seja um máximo da função Lagrangiana associada.

A condição ii) estabelece que para $E$ ser o ótimo, a restrição tem que ser satisfeita e o preço-sombra não pode ser negativo.

A condição iii), usualmente designada por "complementary-slackness condition”, estabelece que o produto do preço-sombra pela restrição é nulo. Esta condição significa que se $\frac{1}{\alpha} \int_{0}^{\infty} e^{-R(t)} E(t) d t-A(0)$ não for ativa (se não for satisfeita na igualdade estrita), então o preço-sombra tem de ser nulo. Se, pelo contrário, o preço-sombra é estritamente positivo, então a restrição associada tem que estar ativa. Em termos econômicos, esta condição estabelece que se a restrição não for ativa e a relaxarmos em uma unidade, daí não decorrerá qualquer alteração na utilidade.

Admitindo que a função objetivo, $U_{t}$, é côncava em $E$ e a restrição faz parte de um conjunto convexo, então as condiçôes necessárias i)-iii) também são suficientes.

Da resolução destas condições retiramos:

$$
\frac{\partial L}{\partial E(t)}=0 \Rightarrow e^{-\rho t} \frac{1}{E(t)}-\frac{\lambda}{\alpha} e^{-R(t)}=0 \Rightarrow e^{-\rho t} \frac{1}{E(t)}=\frac{\lambda}{\alpha} e^{-R(t)}
$$

A condição (A8) estabelece a igualdade entre a utilidade marginal do consumo dos produtos diferenciados, no momento $t$ - tal como é percebida no momento zero -, e o produto da utilidade marginal da riqueza e do valor atualizado do custo de uma unidade do bem no momento $t$.

Resolvendo esta expressão em relação a $E(t)$ e diferenciando-a em relação ao tempo $(t)$ obtemos: 
$\frac{d E(t)}{d t}=\frac{\alpha}{\lambda} e^{R(t)-\rho t}\left(\frac{d R(t)}{d t}-\rho\right) \Rightarrow \dot{E}(t)=\frac{\alpha}{\lambda} e^{R(t)-\rho t}(r-\rho) \Rightarrow \frac{\dot{E}}{E}=r-\rho$

Assim, (A9) evidencia a trajetória temporal ótima do gasto do consumidor representativo [e está representada no texto pela equação (9)].

\section{CONSTRUÇÃO DOS GRÁFICOS 2 E 3}

Dado que $H, L, w_{L} / w_{H}, \rho$ e $a_{H I}$ são constantes, a expressão presenta uma reta horizontal.

$$
\left(\frac{w_{L}}{w_{H}} L+H\right) / \rho a_{H I}+1 \mathrm{re}-
$$

A expressão $\frac{\lambda}{\alpha(\lambda-1)}$ representa uma função com duas assíntotas, uma vertical para $\lambda=1$, outra horizontal ao nível da unidade.

Por último, a expressão $\frac{\lambda}{(\alpha \log \lambda)[\lambda-\alpha(\lambda-1)]}$ representa uma função com assíntota vertical para $\lambda=1$, tendendo para zero quando a dimensão da inovação aumenta indefinidamente $(\lambda \rightarrow+\infty)$.

A posição relativa das curvas $\frac{\lambda}{\alpha(\lambda-1)}$ e $\frac{\lambda}{(\alpha \log \lambda)[\lambda-\alpha(\lambda-1)]}$, ilustradas nos Gráficos 2 e 3 , depende do parâmetro $\alpha$, que representa a proporção do conjunto dos bens diferenciados no índice de consumo do indivíduo representativo. Assim, para $\alpha$ $\leq 0.5$, a primeira curva situa-se sempre $(\forall \lambda)$ à direita da segunda. Quando $\alpha>0.5$, as duas curvas se cruzam num dado $\lambda^{*}>\lambda_{0}$ (com $\lambda_{0}$ representando a dimensão crítica da inovação, isto é, aquela em que $i^{\mathrm{e}}>0$ ). Para $\lambda<\lambda^{*}$, a segunda curva situa-se à direita da primeira, ocorrendo o oposto no caso de $\lambda>\lambda^{*}$.

\section{AS NÃO-CONVEXIDADES E A RELAÇÃO ENTRE TAXA DE INOVAÇÃO DE EQUILÍBRIO E TAXA DE INOVAÇÃO ÓTIMA}

Para avaliar a importância das não-convexidades na relação entre a taxa de inovação de mercado e a taxa de inovação ótima, suponhamos que, num determinado momento $t$, um agente (externo) é bem-sucedido nos seus esforços de pesquisa, dando originem a um avanço tecnológico/melhoria na qualidade na linha de um dado produto $k$. Após o momento $t$, o equilíbrio de mercado virá alterado em $d I$. Na seqüência de tal alteração, o bem-estar dos agentes econômicos restantes também é influenciado. 
Após $t$, a utilidade total dos agentes restantes é, por (37), $U_{t}=\int_{t}^{\infty} e^{-\rho(\tau-t)}\left[(\alpha \log \lambda)(I(\tau)-1)+\log E(\tau)-\alpha \log w_{H}+(1-\alpha) \log \left(\frac{1-\alpha}{\alpha}\right)\right] d \tau$.

O impacto do progresso tecnológico sobre o bem-estar destes agentes (que não o inovador) pode ser medido da seguinte forma:

$$
\frac{d U_{t}}{d I}=\int_{t}^{\infty} e^{-\rho(\tau-t)}(\alpha \log \lambda) d \tau+\int_{t}^{\infty} e^{-\rho(\tau-t)}(1-\alpha) \frac{1}{E(\tau)} \frac{d E(\tau)}{d I} d \tau
$$

O primeiro termo do lado direito da equação (A10) representa dois efeitos: o efeito excedente do consumidor e o efeito spillover intertemporal. O primeiro é o benefício marginal que ocorre para as famílias, após a inovação, e que resulta do fato de estas pagarem pelo produto o mesmo preço que anteriormente, obtendo uma maior quantidade de serviços (spillovers positivos para o consumidor do produto inovador). O segundo representa o benefício, para as gerações futuras, da inovação atual - as gerações futuras irão inovar a partir deste produto de qualidade mais elevada. Desta forma, os dois spillovers positivos gerados pela inovação beneficiam diretamente as famílias.

O segundo termo do lado direito de (A10) representa o efeito destruição do lucro, que se repercute na despesa agregada dos agentes restantes. As inovações provocam perdas nos rendimentos (provenientes dos lucros) destes agentes, o que provoca uma contração nas respectivas despesas. Este spillover negativo que afeta os produtores correntes está associado ao fato do líder destronado perder o fluxo das rendas monopolistas, o que, por sua vez, se traduz numa perda para os proprietários da empresa em questão que, em cada momento do tempo, esperariam receber estas rendas com uma probabilidade 1-idt. Adicionalmente, esta perda de dividendos significa uma menor procura dos outros produtos estado-de-arte, logo, menores lucros para os proprietários das outras empresas.

Com o intuito de determinar o impacto da inovação adicional sobre a despesa agregada, $\frac{d E}{d I}$, recordemos que a despesa agregada iguala o rendimento total menos a poupança, sendo esta, por sua vez, coincidente com o investimento.

Em termos formais,

$$
E(\tau)+Y(\tau)=w_{L} L+w_{H} H+\Pi(\tau)-a_{H I} w_{H} i
$$


em que:

$\Pi(\tau)$ : representa o rendimento-lucro agregado usufruído por todos os atuais produtos estado-de-arte (excluindo o da inovação adicional).

Pela condição de equilíbrio no mercado dos bens, $Y(\tau)=\frac{1-\alpha}{\alpha} E(\tau)$. Donde, $E(\tau)+Y(\tau)=\frac{1}{\alpha} E(\tau)$.

Como se assume que a taxa de melhoria da qualidade (constante) não é afetada pela ocorrência de inovaçóes adicionais no momento $t$, a variação da despesa agregada dos produtos diferenciados, em qualquer momento do tempo $\tau>t$, é igual à variação do rendimento-lucro associado aos produtos diferenciados. Ou seja,

$$
d E(\tau)=\alpha d \Pi(\tau)
$$

Caso não tenha existido uma outra qualquer melhoria na qualidade do produto na indústria $k$ (onde ocorre a inovação) antes do momento $\tau$, a perda de lucros neste preciso momento é $\left(1-\frac{1}{\lambda}\right) E(\tau)$.

Adicionalmente, verifica-se um efeito-multiplicador, pois a redução do lucro na indústria $k$ induz a uma redução na despesa agregada e, em decorrência, uma diminuição nas vendas e no rendimento-lucro das outras indústrias. Incluindo este efeito, a variação no rendimento-lucro agregado é:

$$
\frac{d \Pi(\tau)}{d I(\tau)}=-\left(1-\frac{1}{\lambda}\right) E(\tau)+\left(1-\frac{1}{\lambda}\right) \frac{d E(\tau)}{d I(\tau)}
$$

Como, por $(\mathrm{Al} 2), d \Pi(\tau)=\frac{1}{\alpha} d E(\tau)$, o impacto da inovação adicional sobre a despesa agregada é:

$$
\frac{d E(\tau)}{d I(\tau)}=-\frac{\alpha(\lambda-1)}{\lambda-\alpha(\lambda-1)} E(\tau)
$$

Tendo em conta o processo Poisson subjacente às melhorias na qualidade dos produtos em cada indústria, a probabilidade de não ocorrer qualquer sucesso na pesquisa entre os momentos $t$ e $\tau$ é $e^{-i(\tau-t)}$.

Assim, a redução esperada na despesa agregada num determinado momento $\tau \geq t$, que resulta da inovação adicional ocorrida no momento $t$, é igual a: 


$$
\frac{d E(\tau)}{d I(\tau)}=-E(\tau) \frac{\alpha(\lambda-1)}{\lambda-\alpha(\lambda-1)} e^{-i(\tau-t)}
$$

Substituindo (A15) em (A10) e calculando o integral obtém-se:

$$
\frac{d U_{t}}{d I(\tau)}=\frac{\alpha \log \lambda}{\rho}-\frac{\alpha}{\lambda-\alpha(\lambda-1)} \cdot \frac{\lambda-1}{\rho+i}
$$

ou, de forma equivalente, utilizando (21),

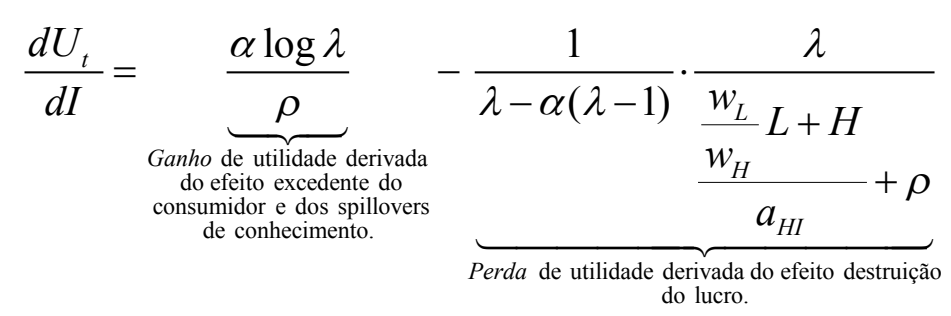

Comparando (47-49), verifica-se que $d U / d I$ é nula quando $i^{e}$ é igual a $i^{*}$, positiva quando $i^{\mathrm{e}}<i^{*}$, e negativa no caso contrário. Assim, confirma-se que a taxa de inovação de equilíbrio é menor que a taxa de inovação ótima quando o efeito combinado do excedente do consumidor e dos spillovers intertemporais domina o efeito destruição do lucro (caso representado nos Gráficos 2 e 3 , neste último, quando $\lambda>\lambda_{1}$ ).

Detalhe das Deduçôes Algébricas Deste Item:

Sendo a utilidade total dada por (37),

$U_{t}=\int_{t}^{\infty} e^{-\rho(\tau-t)}\left[(\alpha \log \lambda)(I(\tau)-1)+\log E(\tau)-\alpha \log w_{H}+(1-\alpha) \log \left(\frac{1-\alpha}{\alpha}\right)\right] d \tau$,

o impacto de uma inovação adicional sobre a utilidade do agente é:

$$
\frac{d U_{t}}{d I(\tau)}=\int_{t}^{\infty} e^{-\rho(\tau-t)}(\alpha \log \lambda) d \tau+\int_{t}^{\infty} e^{-\rho(\tau-t)} \frac{1}{E(\tau)} \frac{d E(\tau)}{d I(\tau)} d \tau .
$$




\section{Cálculo das Integrais}

i) $\int_{t}^{\infty} e^{-\rho(\tau-t)}(\alpha \log \lambda) d \tau=(\alpha \log \lambda) \int_{t}^{\infty} e^{-\rho(\tau-t)} d \tau=-\frac{1}{\rho}(\alpha \log \lambda) \int_{t}^{\infty} e^{-\rho(\tau-t)}(-\rho) d \tau=$

$=\lim _{A \rightarrow+\infty}\left[-\frac{1}{\rho}(\alpha \log \lambda) e^{-\rho(\tau-t)}\right]_{t}^{A}=-\frac{1}{\rho}(\alpha \log \lambda) \lim _{A \rightarrow+\infty} e^{-\rho(A-t)}+\frac{1}{\rho}(\alpha \log \lambda) e^{0}=\frac{\alpha \log \lambda}{\rho}$

ii) $\int_{\mathrm{t}}^{\infty} e^{-\rho(\tau-t)} \frac{1}{E(\tau)} \frac{d E(\tau)}{d I(\tau)} d \tau$

Por (16), tem-se $E(\tau)+Y(\tau)=w_{L} L+w_{H} H+\Pi(\tau)-a_{H I} w_{H} i$

Considerando a condição de equilíbrio do mercado de bens $(8), Y(\tau)=\frac{1-\alpha}{\alpha} E(\tau)$, obtemos $E(\tau)+Y(\tau)=\frac{1}{\alpha} E(\tau)$. E de (16) retiramos que:

$d E(\tau)=\alpha d \pi(\tau) \Rightarrow d \pi(\tau)=\frac{1}{\alpha} d E(\tau)$

Por outro lado, a variação do rendimento-lucro provocada pela inovação adicional é dada por:

$$
\frac{d \Pi(\tau)}{d I(\tau)}=-\left(1-\frac{1}{\lambda}\right) E(\tau)+\left(1-\frac{1}{\lambda}\right) \frac{d E(\tau)}{d I(\tau)} .
$$

Combinando as duas últimas expressões, obtemos a seguinte expressão para o impacto da inovação adicional na despesa agregada:

$\frac{d E(\tau)}{d I(\tau)}=-\frac{\alpha(\lambda-1)}{\lambda-\alpha(\lambda-1)} E(\tau)$

Dadas as características do processo (Processo de Poisson) subjacente às melhorias na qualidade dos produtos, a redução esperada na despesa agregada num determinado momento $\tau \geq t$, resultante da inovação, será: $\frac{d E(\tau)}{d I(\tau)}=-E(\tau) \frac{\alpha(\lambda-1)}{\lambda-\alpha(\lambda-1)} e^{-i(\tau-t)}$ Assim, a integral fica: 


$$
\begin{aligned}
& \int_{\mathrm{t}}^{\infty} e^{-\rho(\tau-t)}(1-\alpha) \frac{1}{E(\tau)} \frac{d E(\tau)}{d I} d \tau=\int_{\mathrm{t}}^{\infty} e^{-\rho(\tau-t)} \frac{1}{E(\tau)}\left[-E(\tau) \frac{\alpha(\lambda-1)}{\lambda-\alpha(\lambda-1)} e^{-i(\tau-t)}\right] d \tau \\
& =-\frac{\alpha(\lambda-1)}{\lambda-\alpha(\lambda-1)} \int_{\mathrm{t}}^{\infty} e^{-(\rho+i)(\tau-t)} d \tau=\frac{1}{(\rho+i)} \frac{\alpha(\lambda-1)}{\lambda-\alpha(\lambda-1)} \int_{\mathrm{t}}^{\infty} e^{-(\rho+i)(\tau-t)}[-(\rho+i)] d \tau \\
& =\frac{1}{(\rho+i)} \frac{\alpha(\lambda-1)}{\lambda-\alpha(\lambda-1)} \lim _{A \rightarrow+\infty}\left[e^{-(\rho+i)(\tau-t)}\right]_{t}^{A}=\frac{1}{(\rho+i)} \frac{\alpha(\lambda-1)}{\lambda-\alpha(\lambda-1)}\left[\lim _{A \rightarrow+\infty} e^{-(\rho+i)(A-t)}-e^{0}\right] \\
& =-\frac{1}{(\rho+i)} \frac{\alpha(\lambda-1)}{\lambda-\alpha(\lambda-1)}=-\frac{(\lambda-1)}{(\rho+i)} \frac{\alpha}{\lambda-\alpha(\lambda-1)} .
\end{aligned}
$$

Como a taxa de inovação de equilíbrio, $i^{\mathrm{e}}$, é dada por (2l), $i^{e}=\frac{\alpha\left(1-\frac{1}{\lambda}\right)\left(\frac{w_{L}}{w_{H}} L+H\right)}{a_{H I}}-\frac{\rho}{\lambda}[\lambda-\alpha(\lambda-1)]$, obtemos:

$$
\int_{\mathrm{t}}^{\infty} e^{-\rho(\tau-t)} \frac{1}{E(\tau)} \frac{d E(\tau)}{d I(\tau)} d \tau=-\frac{\alpha}{\lambda-\alpha(\lambda-1)} \frac{(\lambda-1)}{(\rho+i)}=-\frac{1}{\lambda-\alpha(\lambda-1)} \frac{\lambda}{\rho+\frac{\frac{w_{L}}{w_{H}} L+H}{a_{H I}}} .
$$

Do cálculo das integrais i) e ii) resulta a expressão analítica que nos fornece o impacto da inovação adicional no bem-estar do consumidor, ou seja, a expressão (Al7):

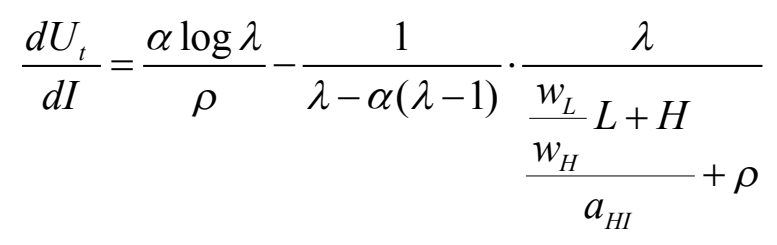

